\title{
Periodic solutions for the Allen-Cahn equation
}

\author{
Rui Huang ${ }^{1,2}$, Haochuan Huang ${ }^{1 *}$, Shanming Ji ${ }^{1}$ and Jingxue Yin ${ }^{1}$
}

"Correspondence:

huanghc@m.scnu.edu.cn

${ }^{1}$ School of Mathematical Sciences,

South China Normal University,

Guangzhou, 510631, China

Full list of author information is

available at the end of the article

\begin{abstract}
In this paper we consider the time-periodic Allen-Cahn equation subject to homogeneous boundary value condition and time-periodic condition. For the case of a smooth bounded domain with spatial dimension $N \leq 3$, we prove the existence of classical nontrivial periodic solutions. For the case of a star shaped domain with $N \geq 4$, we prove the nonexistence of nontrivial periodic solutions. For the case of an annulus domain with $N \geq 3$, we prove the existence of nontrivial radial periodic solutions. Some numerical simulations are also presented to illustrate our results.
\end{abstract}

Keywords: periodic solutions; existence; nonexistence; numerical simulations

\section{Introduction}

This paper is concerned with the following time-periodic Allen-Cahn equation:

$$
\begin{aligned}
& \frac{\partial u}{\partial t}-\Delta u=m(t)\left(u^{3}-u\right), \quad x \in \Omega, t \in \mathbb{R}^{+}, \\
& u(x, t)=0, \quad x \in \partial \Omega, t \in \mathbb{R}^{+}, \\
& u(x, t+\omega)=u(x, t)>0, \quad x \in \Omega, t \in \mathbb{R}^{+},
\end{aligned}
$$

where $\Omega \subset \mathbb{R}^{N}$ is a domain, $\omega$ is a positive constant, $m(t)$ is a positive $\omega$-periodic function. This problem has its origin in the gradient theory of phase transitions, which describes the motion of antiphase boundaries in crystalline solids; the process of phase separation in iron alloys, including order-disorder transitions; see for example [1-3] and references therein. It is also well accepted that the Allen-Cahn equations can be used to describe population dynamics; see for example [4]. Since the pioneering work of Allen and Cahn [1], a large and still-growing body of work is concerned with the study of the Allen-Cahn equations in different aspects; see [2, 3, 5, 6] etc. Among them, the study of Allen-Cahn type equations with spatial periodicity has attracted much attention; see for example [711] and references therein. However, as far as we know, there are few investigations concerned with the time-periodic solutions of Allen-Cahn type equations. Here, inspired by the ideas described in $[4,12]$, we give a sketch of the formulation of (1.1) from modeling the growth and dispersal in the population which is sensitive to time-periodic factors. Let $u(x, t)$ be the population density. If the population is sensitive to the environment, just as described in [4], it is reasonable to suppose that the energy maintains a spatial heterogene-

(c) 2015 Huang et al. This article is distributed under the terms of the Creative Commons Attribution 4.0 International License (http://creativecommons.org/licenses/by/4.0/), which permits unrestricted use, distribution, and reproduction in any medium, provided you give appropriate credit to the original author(s) and the source, provide a link to the Creative Commons license, and indicate if changes were made. 
ity depending on the neighboring gradient in the population density, and the total energy $F[u]$ in the region $\Omega$ occupied by the population is given by

$$
F[u]=\int_{\Omega}\left(\frac{1}{2}|\nabla u|^{2}+H(u, t)\right) d x
$$

The formal variation $\delta F[u]$ with respect to $u$ that vanishes on $\partial \Omega$ is given by

$$
\begin{aligned}
\delta F[u] & =\int_{\Omega}\left(\nabla u \cdot \delta \nabla u+\frac{\partial H(u, t)}{\partial u} \delta u\right) d x \\
& =\int_{\Omega}\left(-\Delta u+\frac{\partial H(u, t)}{\partial u}\right) \delta u d x .
\end{aligned}
$$

Then we have the following expression:

$$
\frac{\delta F}{\delta u}=-\Delta u+\frac{\partial H(u, t)}{\partial u}
$$

for the variational derivative. Equilibrium is characterized by the vanishing of $\frac{\delta F}{\delta u}$; the hypothesis underlying the standard derivation is that relaxation toward equilibrium is governed by the following relation:

$$
\frac{\partial u}{\partial t}=-\frac{\delta F}{\delta u}=\Delta u-\frac{\partial H(u, t)}{\partial u} .
$$

In particular, if the population is sensitive to seasons, then it is reasonable to investigate the existence of time-periodic solutions of (1.4). From this motivation, in the present paper, we are interested in considering the following internal energy density:

$$
H(u, t)=-\frac{1}{4} m(t)\left(u^{2}-1\right)^{2},
$$

which combined with (1.4) yields (1.1).

Since the last century, periodic parabolic equations have been the subject of extensive study; see for example [13-22] and the references therein. Among the earliest works of this aspect, we refer to Esteban [13], in which the author considered the following equation:

$$
\frac{\partial u}{\partial t}-\Delta u=m(t) u^{q}
$$

and proved the existence of positive periodic classical solutions for the case of $1<q<$ $(N+1) /(N-1)$ under the assumption

$$
m(t+\omega)=m(t), \quad m \in W^{1, \infty}[0, \omega], \quad \inf _{t \in[0, \omega]} m(t)=m_{0}>0 .
$$

She also proved that, for the case of $1<q<(3 N+8) /(3 N-4)$, under an additional technical assumption on $m(t)$, the existence of positive periodic classical solutions is also true. Later, Esteban improved her results in [14] and proved the existence of positive periodic classical solutions for any $q>1$ with $N \leq 2$, and for $1<q<N /(N-2)$ with $N>2$ for any smooth 
positive $m(t)$. Then Quittner [18] obtained the existence results with the optimal growth assumption on $q$, i.e. $1<q<q_{S}$, where

$$
q_{S}:=\frac{N+2}{N-2} \quad \text { if } N>2, \quad q_{S}:=+\infty \quad \text { if } N \leq 2 .
$$

Moreover, Húska $[15,16]$ considered a more general equation

$$
\frac{\partial u}{\partial t}-\Delta u=m(t) f(x, u)
$$

and obtained the existence of positive periodic solutions under some structural assumptions on $m(t)$ and $f(x, u)$. Recently, Yin and Jin [21] considered the $p$-Laplacian

$$
\frac{\partial u}{\partial t}-\operatorname{div}\left(|\nabla u|^{p-2} \nabla u\right)=m(x, t) u^{q}, \quad p>1, q \geq 0,
$$

which may have degeneracy or singularity. They give a rather complete characterization in terms of the parameter $p$ and the exponent $q$, of whether or not the positive periodic solutions exist. Later, Deng [23] considered a radial problem for the $p$-Laplacian (1.5) in annulus domain $\Omega=B_{R_{0}} \backslash B_{r_{0}}$, and give the existence of radial periodic solutions provided that $q \geq \frac{N p}{N-p}-1$ with $N>p$.

To the best of our knowledge, there is little literature about nodal periodic solutions (that is, periodic solutions may change sign) except for some special cases. For example, when $\Omega=B_{R}:=\left\{x \in \mathbb{R}^{N}:|x|<R\right\}$, Bartsch et al. ([24], Theorem 1.8) obtained the existence of radial time-periodic solutions with a prescribed number of spatial sign changes. In the population dynamics model, the species is restricted to the bounded heterogeneous environment $\Omega$ whose boundary is prohibitive to the species. In this paper, we are interested in the case of constant-sign solutions. Without loss of generality, we consider the positive periodic solutions throughout the whole paper.

Even though there already exist some well-known results on semilinear parabolic PDEs with initial boundary value conditions, as far as we know, there are few references related to the Allen-Cahn type equation with time-periodic condition. To describe the population dynamics which is sensitive to time-periodic factors (for instance seasons etc.), in the present paper, we investigate the time-periodic Allen-Cahn type problem (1.1)-(1.3). There are also some results on periodic parabolic equations with Neumann boundary conditions (see [25-28] for example). For the Allen-Cahn equation, the homogenous Neumann boundary conditions imply that no mass loss occurs across the boundary walls (cf. [3], p.2). From a biological point of view, the homogenous Neumann boundary conditions model the trend of the species to survive on the boundary, while the homogenous Dirichlet boundary conditions are used to describe that the boundary is lethal to the species (see $[26,28])$. Obviously, the Neumann boundary value conditions will cause some additional difficulties in establishing the a priori estimates (see for instance $[26,28]$ ), which will be the topic of our further work in the future as well as the corresponding problems with Dirichlet-Neumann boundary conditions.

This paper is organized as follows. In Section 2, as a preparation, we introduce some notations and state the main results. In Section 3, we prove $\operatorname{deg}\left(I-K_{1}, B_{r}, 0\right)=1$. In Section 4, we first obtain a Pohozaev type identity and the $W_{\omega}^{1,1 ; 2}\left(Q_{\omega}\right)$ estimates. We then base on interpolation estimates and Morse-type iteration to obtain $L_{\omega}^{\infty}\left(Q_{\omega}\right)$ estimates. Eventually, we 
use $L_{\omega}^{\infty}\left(Q_{\omega}\right)$ estimates to prove $\operatorname{deg}\left(I-K_{1}, B_{R}, 0\right)=0$. In Section 5 , we consider the problem (1.1)-(1.3) in an annulus domain $\Omega=B_{R_{0}} \backslash B_{r_{0}}$. In order to calculate $\operatorname{deg}\left(I-T_{l}, B_{\widehat{R}}, 0\right)$, we will obtain the $L_{\omega}^{\infty}\left(\left[s_{0}, s_{1}\right] \times[0, \omega]\right)$ estimates for radial solutions. Thereafter, we devote our work to proving the main results of this paper in Section 6. Finally, in Section 7, numerical simulations are provided to illustrate how the positive periodic solution of problem (1.1)-(1.3) depends on changing $m(t)$.

\section{Preliminaries and the main results}

In this section we give some preliminaries and present the main results of this paper. The following notations will be used throughout the paper.

$Q_{\omega}=\Omega \times(0, \omega), \inf _{t \in[0, \omega]} m(t):=m_{0}>0,\|m\|_{L^{\infty}[0, \omega]}:=M ;$

$N$ : the dimension of space;

$C$ : positive constant independent of $u$, though $C$ may vary from step to step;

$B_{R}$ : a ball in $L_{\omega}^{\infty}\left(Q_{\omega}\right)$ with center zero and radius $R$;

$\operatorname{deg}\left(I-K_{\lambda}, B_{R}, 0\right)$ : the Leray-Schauder degree of a compact operator $I-K_{\lambda}$ in $B_{R}$ with respect to 0 ;

$$
\begin{aligned}
& L_{\omega}^{\infty}\left(Q_{\omega}\right):=\left\{u(x, t)=u(x, t+\omega) \mid u \in L^{\infty}\left(Q_{\omega}\right)\right\} ; \\
& C_{\omega}^{2+\alpha, 1+\frac{\alpha}{2}}\left(Q_{\omega}\right):=\left\{u(x, t)=u(x, t+\omega) \mid u \in C^{2+\alpha, 1+\frac{\alpha}{2}}\left(Q_{\omega}\right), 0<\alpha<1\right\} .
\end{aligned}
$$

The following hypotheses will be used in our proof.

$\left(\mathrm{H}_{1}\right) m(t+\omega)=m(t), m \in W^{1, \infty}[0, \omega], \inf _{t \in[0, \omega]} m(t)=m_{0}>0$,

$\left(\mathrm{H}_{2}\right) \sup _{t \in[0, \omega]} \frac{\left(m^{\prime}(t)\right)^{-}}{m(t)}<\frac{2}{R^{2}(\Omega)}$,

where $\left(m^{\prime}(t)\right)^{-}=\max \left\{0,-m^{\prime}(t)\right\}, R(\Omega)=\sup _{x \in \Omega}|x|$.

The main results of this paper are the following theorems.

Theorem 2.1 Suppose the assumption $\left(\mathrm{H}_{1}\right)$ holds, and $\Omega$ is a smooth bounded convex domain. If $N \leq 2$, then the problem (1.1)-(1.3) admits a nontrivial classical periodic solution $u \in C_{\omega}^{2+\alpha, 1+\frac{\alpha}{2}}\left(Q_{\omega}\right)$. If $N=3$, under an additional assumption $\left(\mathrm{H}_{2}\right)$, then the problem (1.1)(1.3) also admits a nontrivial classical periodic solution $u \in C_{\omega}^{2+\alpha, 1+\frac{\alpha}{2}}\left(Q_{\omega}\right)$.

Theorem 2.2 If $N \geq 4$ and $\Omega$ is star shaped, then the problem (1.1)-(1.3) does not admit nontrivial periodic solutions.

Theorem 2.3 Suppose the assumption $\left(\mathrm{H}_{1}\right)$ holds. If $N \geq 3$ and $\Omega=B_{R_{0}} \backslash B_{r_{0}}$ is an annulus domain, then the problem (1.1)-(1.3) admits a nontrivial radial periodic solutions $u \in C_{\omega}^{2+\alpha, 1+\frac{\alpha}{2}}\left(Q_{\omega}\right)$.

Remark 2.1 Our result of Theorem 2.3 is different from Deng ([23], Theorem 3.1), where the existence of nontrivial radial periodic solutions for the $p$-Laplacian (1.5) relies on the condition of $q \geq \frac{N p}{N-p}-1$ with $N>p$. However, for the case of $N=3$ and $p=2$, we find that for $q=3$ the problem (1.1)-(1.3) also admits nontrivial radial periodic solutions. That means the source $-m(t) u$ indeed makes some sense in the present problem.

In order to employ the topological method to deal with the existence of the nontrivial periodic solutions of the problem (1.1)-(1.3), we introduce the operators $K_{\lambda}$ from $L_{\omega}^{\infty}\left(Q_{\omega}\right)$ 
to itself,

$$
\begin{aligned}
& K_{\lambda}: L_{\omega}^{\infty}\left(Q_{\omega}\right) \times[0,1] \rightarrow L_{\omega}^{\infty}\left(Q_{\omega}\right), \\
& (u, \lambda) \mapsto v .
\end{aligned}
$$

We say that $K_{\lambda} u=v$, if $v$ is a solution of the following problem:

$$
\left\{\begin{array}{l}
\frac{\partial v}{\partial t}-\Delta v=\lambda m(t)\left(u^{3}-u\right), \quad \lambda \in[0,1], x \in \Omega, t \in(0, \omega) \\
v(x, t)=0, \quad x \in \partial \Omega, t \in(0, \omega) \\
v(x, 0)=v(x, \omega), \quad x \in \Omega .
\end{array}\right.
$$

By classical linear theory, we can easily check that the following lemma is true.

Lemma 2.1 The operators $K_{\lambda}$ is well defined and completely continuous.

$3 \operatorname{deg}\left(I-K_{1}, B_{r}, 0\right)=1$

Obviously, the periodic solutions for the problem (2.1) are fixed points of the operator $K_{\lambda}$ from $L_{\omega}^{\infty}\left(Q_{\omega}\right)$ to itself. We first investigate the Leray-Schauder degree of $I-K_{\lambda}$ in a small ball $B_{r}$ in $L_{\omega}^{\infty}\left(Q_{\omega}\right)$.

We denote by $\lambda_{1}$ the first eigenvalue of $-\Delta$ in $H_{0}^{1}(\Omega)$, namely

$$
\left\{\begin{array}{l}
-\Delta u=\lambda u, \quad x \in \Omega \\
u=0, \quad x \in \partial \Omega
\end{array}\right.
$$

where $\lambda_{1}$ is given by the following formula:

$$
\lambda_{1}=\inf _{u \neq 0, u \in H_{0}^{1}(\Omega)} \frac{\int_{\Omega}|\nabla u|^{2} d x}{\int_{\Omega} u^{2} d x} .
$$

We then have

$$
\lambda_{1} \int_{\Omega} u^{2} d x \leq \int_{\Omega}|\nabla u|^{2} d x
$$

We denote $\varphi_{1}(x)$ the corresponding eigenfunction, namely

$$
\left\{\begin{array}{l}
-\Delta \varphi_{1}(x)=\lambda_{1} \varphi_{1}(x), \quad x \in \Omega, \\
\varphi_{1}(x)=0, \quad x \in \partial \Omega .
\end{array}\right.
$$

Proposition $3.1 \operatorname{deg}\left(I-K_{1}, B_{r}, 0\right)=1$.

Proof We argue by contradiction to prove that $K_{\lambda}$ has no fixed points on $\partial B_{r}$, namely

$$
K_{\lambda} u \neq u, \quad \lambda \in[0,1], u \in \partial B_{r} .
$$

If $\lambda=0, K_{0}$ is null operator, then

$$
K_{0}(u)=0 \neq u, \quad u \in \partial B_{r} .
$$


Thus (3.4) holds. We need only to consider the remaining case of $0<\lambda \leq 1$. Suppose that the operator $K_{\lambda}$ admits fixed points when $u \in \partial B_{r} \subset L_{\omega}^{\infty}\left(Q_{\omega}\right)$ for some $\lambda \in(0,1]$. Replacing $v$ with $u$ in the first equation of (2.1), we have

$$
\frac{\partial u}{\partial t}=\Delta u+\lambda m(t)\left(u^{3}-u\right), \quad \lambda \in(0,1]
$$

We choose $r$ sufficiently small, such that

$$
0<r<\left(\frac{\lambda_{1}}{M}\right)^{\frac{1}{2}}, \quad r=\|u\|_{L_{\omega}^{\infty}\left(Q_{\omega}\right)}
$$

where $\lambda_{1}$ is defined in (3.1). Multiplying (3.5) by $u$, integrating over $Q_{\omega}$, using (3.2) and (3.6), by the time periodicity of $u$, we obtain

$$
\begin{aligned}
0 & =\iint_{Q_{\omega}} \frac{\partial u}{\partial t} u d x d t \\
& =-\iint_{Q_{\omega}}|\nabla u|^{2} d x d t+\lambda \iint_{Q_{\omega}} m(t) u^{4} d x d t-\lambda \iint_{Q_{\omega}} m(t) u^{2} d x d t \\
& \leq-\lambda_{1} \iint_{Q_{\omega}} u^{2} d x d t+\|m(t)\|_{L^{\infty}[0, \omega]} \iint_{Q_{\omega}}\|u\|_{L_{\omega}^{\infty}\left(Q_{\omega}\right)}^{2} u^{2} d x d t \\
& =\left(M r^{2}-\lambda_{1}\right) \iint_{Q_{\omega}} u^{2} d x d t \\
& <0,
\end{aligned}
$$

which is a contradiction. Therefore $K_{\lambda}$ has no fixed points on $\partial B_{r}$, then (3.4) holds. On the other hand, since the operator $K_{0}=0$, using the homotopy invariance of the LeraySchauder degree, we have

$$
\operatorname{deg}\left(I-K_{1}, B_{r}, 0\right)=\operatorname{deg}\left(I-K_{0}, B_{r}, 0\right)=\operatorname{deg}\left(I, B_{r}, 0\right)=1 .
$$

The proof of this proposition is complete.

$4 \operatorname{deg}\left(I-K_{1}, B_{R}, 0\right)=0$

In this section we investigate the Leray-Schauder degree of $I-K_{\lambda}$ in a large ball $B_{R}$ in $L_{\omega}^{\infty}\left(Q_{\omega}\right)$. Let us now introduce a new family of operators $T_{L}$,

$$
\begin{aligned}
& T_{L}: L_{\omega}^{\infty}\left(Q_{\omega}\right) \times\left[0, L_{0}\right] \rightarrow L_{\omega}^{\infty}\left(Q_{\omega}\right), \\
& (u, L) \mapsto v,
\end{aligned}
$$

where the constant $L_{0}>1+\frac{\lambda_{1}}{m_{0}}$, and $\lambda_{1}$ is defined in (3.1). We say that $T_{L} u=v$, if $v$ is a solution of the following problem:

$$
\left\{\begin{array}{l}
\frac{\partial v}{\partial t}-\Delta v=m(t)\left(u^{3}+u+L u\right), \quad L \in\left[0, L_{0}\right], x \in \Omega, t \in(0, \omega) \\
v(x, t)=0, \quad x \in \partial \Omega, t \in(0, \omega) \\
v(x, 0)=v(x, \omega), \quad x \in \Omega
\end{array}\right.
$$


We can easily check that $T_{L}$ is well defined and completely continuous. The fixed point of $T_{L}$ solves the following problem:

$$
\left\{\begin{array}{l}
\frac{\partial u}{\partial t}-\Delta u=m(t)\left(u^{3}-u+L u\right), \quad L \in\left[0, L_{0}\right], x \in \Omega, t \in(0, \omega), \\
u(x, t)=0, \quad x \in \partial \Omega, t \in(0, \omega), \\
u(x, 0)=u(x, \omega), \quad x \in \Omega .
\end{array}\right.
$$

Lemma 4.1 There exists a positive constant $L_{0}>1+\frac{\lambda_{1}}{m_{0}}$ such that

$$
T_{L} u \neq u, \quad L \geq L_{0} .
$$

Proof We argue by contradiction. Suppose that the operator $T_{L}$ admits fixed points for $L \geq L_{0}$. Multiplying the first equation of (4.2) by $\varphi_{1}(x)$, where $\varphi_{1}(x)$ is defined in (3.3), integrating over $Q_{\omega}$, and using the periodicity of $u$, we have

$$
\begin{gathered}
\underbrace{\iint_{Q_{\omega}} \frac{\partial u}{\partial t} \varphi_{1}(x) d x d t}_{0}-\iint_{Q_{\omega}} \Delta u \varphi_{1}(x) d x d t \\
=\iint_{Q_{\omega}} m(t)\left(u^{2}-1+L\right) \varphi_{1}(x) u d x d t .
\end{gathered}
$$

Now we calculate the second term on the left-hand side of (4.3). By (3.3), we obtain

$$
\begin{aligned}
-\iint_{Q_{\omega}} \Delta u \varphi_{1}(x) d x d t & =\iint_{Q_{\omega}}\left(-\Delta \varphi_{1}(x)\right) u d x d t \\
& =\iint_{Q_{\omega}} \lambda_{1} \varphi_{1}(x) u d x d t .
\end{aligned}
$$

Substituting (4.4) into (4.3), we have

$$
\iint_{Q_{\omega}} \lambda_{1} \varphi_{1}(x) u d x d t=\iint_{Q_{\omega}} m(t)\left(u^{2}-1+L\right) \varphi_{1}(x) u d x d t
$$

If $L \geq L_{0}>1+\frac{\lambda_{1}}{m_{0}}, u>0, x \in \Omega$, using (4.5), we obtain

$$
\begin{aligned}
& \iint_{Q_{\omega}} m(t)\left(u^{2}-1+L\right) \varphi_{1}(x) u d x d t \\
& \quad \geq \inf _{t \in[0, \omega]} m(t) \iint_{Q_{\omega}}\left(u^{2}-1+L\right) \varphi_{1}(x) u d x d t \\
& \quad>m_{0} \iint_{Q_{\omega}}\left(u^{2}+\frac{\lambda_{1}}{m_{0}}\right) \varphi_{1}(x) u d x d t \\
& \quad>\iint_{Q_{\omega}} \lambda_{1} \varphi_{1}(x) u d x d t
\end{aligned}
$$

which is contradict with (4.5). Thus $T_{L}$ has no fixed points for $L \geq L_{0}$. The proof of this lemma is complete. 
Similar to the proof of Lemma 10 in [13] and a detailed proof of Theorem 4.2.1 in [16], we have the following lemma.

Lemma 4.2 Let $\Omega \subset \mathbb{R}^{N}$ is a smooth and convex bounded domain. If $m(t)$ satisfies the assumption $\left(\mathrm{H}_{1}\right)$, then there exist constant $C>0$ and $\varepsilon>0(C, \varepsilon$ independent of $L, L \in$ $\left.\left[0, L_{0}\right]\right)$, such that if $u$ is a solution of the problem (4.2), then

$$
\begin{aligned}
& \sup _{\Omega_{\varepsilon} \times[0, \omega]}|u(x, t)| \leq C, \\
& \sup _{\Omega_{\varepsilon} \times[0, \omega]}|\nabla u(x, t)| \leq C, \quad(x, t) \in \bar{\Omega}_{\varepsilon} \times[0, \omega],
\end{aligned}
$$

where $\Omega_{\varepsilon}=\{x \in \Omega ; \operatorname{dist}(x, \partial \Omega)<\varepsilon\}$

Lemma 4.3 Suppose the assumption $\left(\mathrm{H}_{1}\right)$ holds. If $u$ is a classical solution of the problem (4.2), then

$$
\begin{aligned}
& \iint_{Q_{\omega}}\left(\left(1-\frac{N}{4}\right) u^{4}-u^{2}+L u^{2}\right) m(t) d x d t \\
& =\iint_{Q_{\omega}}\left(m^{\prime}(t)\left(\frac{u^{4}}{4}-\frac{u^{2}}{2}+\frac{L u^{2}}{2}\right)+\left(u_{t}\right)^{2}\right) \frac{|x|^{2}}{2} d x d t \\
& \quad+\frac{1}{2} \iint_{\partial \Omega \times(0, \omega)}|\nabla u|^{2}(x \cdot \vec{n}) d s d t .
\end{aligned}
$$

Proof Multiplying the first equation of (4.2) by $(x \cdot \nabla u)$, and integrating over $Q_{\omega}$, we have

$$
\begin{gathered}
\iint_{Q_{\omega}} u_{t}(x \cdot \nabla u) d x d t-\iint_{Q_{\omega}} \Delta u(x \cdot \nabla u) d x d t \\
\quad=\iint_{Q_{\omega}} m(t)\left(u^{3}-u+L u\right)(x \cdot \nabla u) d x d t .
\end{gathered}
$$

Multiplying the first equation of (4.2) by $u_{t} \frac{|x|^{2}}{2}$, and integrating over $Q_{\omega}$, using the periodicity of $u$, we obtain

$$
\begin{aligned}
& \iint_{Q_{\omega}}\left(u_{t}\right)^{2} \frac{|x|^{2}}{2} d x d t-\iint_{Q_{\omega}} \Delta u\left(u_{t} \frac{|x|^{2}}{2}\right) d x d t \\
& \quad=\iint_{Q_{\omega}} m(t)\left(\left(u^{3}-u+L u\right) u_{t}\right) \frac{|x|^{2}}{2} d x d t \\
& \quad=\iint_{Q_{\omega}} m(t) \frac{\partial\left(\frac{u^{4}}{4}-\frac{u^{2}}{2}+\frac{L u^{2}}{2}\right)}{\partial t} \frac{|x|^{2}}{2} d x d t \\
& \quad=-\iint_{Q_{\omega}} m^{\prime}(t)\left(\frac{u^{4}}{4}-\frac{u^{2}}{2}+\frac{L u^{2}}{2}\right) \frac{|x|^{2}}{2} d x d t .
\end{aligned}
$$

Now we calculate the second term on the left side of (4.8). By (1.2), we have

$$
u_{t}(x, t)=0, \quad x \in \partial \Omega .
$$


Integrating by parts over $Q_{\omega}$, using the periodicity of $u$ and (4.9), we obtain

$$
\begin{aligned}
& \iint_{Q_{\omega}} \Delta u\left(u_{t} \frac{|x|^{2}}{2}\right) d x d t \\
& =\underbrace{\int_{0}^{\omega} \int_{\partial \Omega}(\nabla u \cdot \vec{n}) u_{t} \frac{|x|^{2}}{2} d s d t}_{0}-\iint_{Q_{\omega}} \sum_{i=1}^{N} u_{x_{i}}\left(u_{t x_{i}} \frac{|x|^{2}}{2}+u_{t} x_{i}\right) d x d t \\
& =-\underbrace{-\iint_{Q_{\omega}} \sum_{i=1}^{N} \frac{d}{d t}\left(\frac{u_{x_{i}}^{2}}{2}\right) \frac{|x|^{2}}{2} d x d t}_{0}-\iint_{Q_{\omega}} u_{t} \sum_{i=1}^{N} x_{i} u_{x_{i}} d x d t \\
& =-\iint_{Q_{\omega}} u_{t}(x \cdot \nabla u) d x d t .
\end{aligned}
$$

Substituting (4.10) into (4.8), we have

$$
\begin{aligned}
& \iint_{Q_{\omega}}\left(u_{t}\right)^{2} \frac{|x|^{2}}{2} d x d t+\iint_{Q_{\omega}} u_{t}(x \cdot \nabla u) d x d t \\
& \quad=-\iint_{Q_{\omega}} m^{\prime}(t)\left(\frac{u^{4}}{4}-\frac{u^{2}}{2}+\frac{L u^{2}}{2}\right) \frac{|x|^{2}}{2} d x d t .
\end{aligned}
$$

By (4.7) and (4.11), we have

$$
\begin{array}{r}
\iint_{Q_{\omega}}\left(u_{t}\right)^{2} \frac{|x|^{2}}{2} d x d t+\iint_{Q_{\omega}} \Delta u(x \cdot \nabla u) d x d t \\
=-\iint_{Q_{\omega}} m^{\prime}(t)\left(\frac{u^{4}}{4}-\frac{u^{2}}{2}+\frac{L u^{2}}{2}\right) \frac{|x|^{2}}{2} d x d t \\
\quad-\iint_{Q_{\omega}} m(t)\left(u^{3}-u+L u\right)(x \cdot \nabla u) d x d t .
\end{array}
$$

Now we calculate the second term on the right-hand side of (4.12). We recall the divergence theorem,

$$
\int_{\partial \Omega} \vec{w} \cdot \vec{n} d s=\int_{\Omega} \operatorname{div} \vec{w} d x
$$

where $\vec{n}$ is the unit outward normal vector at $x \in \partial \Omega, d s$ is the surface area element of $\partial \Omega$. We choose $\vec{w}=\left(\frac{u^{4}}{4}-\frac{u^{2}}{2}+\frac{L u^{2}}{2}\right) x$. By the second equation of (4.2), we have

$$
\begin{aligned}
0= & \iint_{\partial \Omega \times(0, \omega)} m(t)\left(\frac{u^{4}}{4}-\frac{u^{2}}{2}+\frac{L u^{2}}{2}\right) x \cdot \vec{n} d s d t \\
= & \iint_{Q_{\omega}} m(t) \operatorname{div}\left(\left(\frac{u^{4}}{4}-\frac{u^{2}}{2}+\frac{L u^{2}}{2}\right) x\right) d x d t \\
= & N \iint_{Q_{\omega}} m(t)\left(\frac{u^{4}}{4}-\frac{u^{2}}{2}+\frac{L u^{2}}{2}\right) d x d t \\
& +\iint_{Q_{\omega}} m(t)\left(u^{3}-u+L u\right)(x \cdot \nabla u) d x d t .
\end{aligned}
$$


In the following, we calculate the second term on the left side of (4.12). Multiplying the first equation of (4.2) by $u$, integrating over $Q_{\omega}$, and using the periodicity of $u$, we obtain

$$
\underbrace{\iint_{Q_{\omega}} \frac{\partial u}{\partial t} u d x d t}_{0}+\iint_{Q_{\omega}}|\nabla u|^{2} d x d t=\iint_{Q_{\omega}} m(t)\left(u^{4}-u^{2}+L u^{2}\right) d x d t .
$$

We denote $D_{i}=\frac{\partial u}{\partial x_{i}}, D_{i j}=\frac{\partial}{\partial x_{j}}\left(\frac{\partial u}{\partial x_{i}}\right)$, choose $\vec{w}=|\nabla u|^{2} x$, and use (4.13) to get

$$
\begin{aligned}
& \iint_{\partial \Omega \times(0, \omega)}\left(|\nabla u|^{2} x\right) \cdot \vec{n} d s d t \\
& =\iint_{Q_{\omega}} \operatorname{div}\left(|\nabla u|^{2} x\right) d x d t \\
& =N \iint_{Q_{\omega}}|\nabla u|^{2} d x d t+\iint_{Q_{\omega}} \sum_{i=1}^{N} x_{i} D_{i}\left(\sum_{j=1}^{N}\left|D_{j} u\right|^{2}\right) d x d t \\
& =N \iint_{Q_{\omega}}|\nabla u|^{2} d x d t+2 \iint_{Q_{\omega}} \sum_{j=1}^{N} D_{j} u\left(\sum_{i=1}^{N} x_{i} D_{i j} u\right) d x d t \\
& =N \iint_{Q_{\omega}}|\nabla u|^{2} d x d t-2 \iint_{Q_{\omega}} \sum_{j=1}^{N} u D_{j}\left(\sum_{i=1}^{N} x_{i} D_{i j} u\right) d x d t \\
& =N \iint_{Q_{\omega}}|\nabla u|^{2} d x d t-2 \iint_{Q_{\omega}} u \Delta u d x d t-2 \iint_{Q_{\omega}} \sum_{i=1}^{N}\left(u x_{i}\right) D_{i}(\Delta u) d x d t \\
& =N \iint_{Q_{\omega}}|\nabla u|^{2} d x d t-2 \iint_{Q_{\omega}} u \Delta u d x d t+2 \iint_{Q_{\omega}} \sum_{i=1}^{N} D_{i}\left(u x_{i}\right)(\Delta u) d x d t \\
& =N \iint_{Q_{\omega}}|\nabla u|^{2} d x d t-2 \iint_{Q_{\omega}} u \Delta u d x d t \\
& \quad+2 N \iint_{Q_{\omega}} u \Delta u d x d t+2 \iint_{Q_{\omega}} \Delta u(x \cdot \nabla u) d x d t \\
& =(2-N) \iint_{Q_{\omega}}|\nabla u|^{2} d x d t+2 \iint_{Q_{\omega}} \Delta u(x \cdot \nabla u) d x d t .
\end{aligned}
$$

Substituting (4.15) into the above equality, we have

$$
\begin{aligned}
& \iint_{Q_{\omega}} \Delta u(x \cdot \nabla u) d x d t \\
& =\frac{(N-2)}{2} \iint_{Q_{\omega}}|\nabla u|^{2} d x d t+\frac{1}{2} \iint_{\partial \Omega \times(0, \omega)}|\nabla u|^{2}(x \cdot \vec{n}) d s d t \\
& =\frac{(N-2)}{2} \iint_{Q_{\omega}} m(t)\left(u^{4}-u^{2}+L u^{2}\right) d x d t \\
& \quad+\frac{1}{2} \iint_{\partial \Omega \times(0, \omega)}|\nabla u|^{2}(x \cdot \vec{n}) d s d t .
\end{aligned}
$$

Substituting (4.14) and (4.16) into (4.12), we obtain the so-called Pohozaev-identity (4.6). The proof of this lemma is complete. 
Lemma 4.4 For $N \leq 2$, suppose the assumption $\left(\mathrm{H}_{1}\right)$ holds, if $u$ is a solution of the problem (4.2), then

$$
\|u\|_{W_{\omega}^{11,1 ;}\left(Q_{\omega}\right)}^{2}=\iint_{Q_{\omega}}|\nabla u|^{2}+u_{t}^{2} d x d t \leq C,
$$

where the positive constant $C$ is independent of $L$ and $u$.

Proof Multiplying the first equation of (4.2) by $\varphi_{1}(x)$, integrating over $Q_{\omega}$, using (3.3), we get

$$
\iint_{Q_{\omega}} \frac{\partial u}{\partial t} \varphi_{1}(x) d x d t+\lambda_{1} \iint_{Q_{\omega}} u \varphi_{1}(x) d x d t=\iint_{Q_{\omega}} m(t)\left(u^{3}-u+L u\right) \varphi_{1}(x) d x d t
$$

Using the periodicity of $u$ and (4.17), for $L \in\left[0, L_{0}\right]$, we have

$$
\iint_{Q_{\omega}} m(t) u^{3} \varphi_{1}(x) d x d t=\iint_{Q_{\omega}}\left(\lambda_{1}+m(t)-\operatorname{Lm}(t)\right) u \varphi_{1}(x) d x d t
$$

We shall use the fact that if $\Omega$ is of class $C^{2}$, then there exist constants $c_{1}, c_{2}>0$, such that

$$
c_{1} \operatorname{dist}(x, \partial \Omega) \leq \varphi_{1}(x) \leq c_{2} \operatorname{dist}(x, \partial \Omega), \quad x \in \Omega
$$

This is a consequence of $u \in C^{1}(\bar{\Omega})$ and of Hopf's lemma (see Proposition 52.1(iii) in [29] for example). Since $\Omega$ is bounded, we have

$$
\operatorname{dist}(x, \partial \Omega) \leq R(\Omega) .
$$

By the assumption $\left(\mathrm{H}_{1}\right)$, we have $0<m_{0} \leq m(t) \leq M$. Using Hölder's inequality, (4.18) and (4.19), we have

$$
\begin{aligned}
& m_{0} \iint_{Q_{\omega}} u^{3} \varphi_{1}(x) d x d t \\
& \quad \leq \iint_{Q_{\omega}}\left(\lambda_{1}+m(t)\right) u \varphi_{1}(x) d x d t \\
& \quad \leq\left(\lambda_{1}+M\right) \iint_{Q_{\omega}}\left(u \varphi_{1}^{\frac{1}{3}}\right) \varphi_{1}^{\frac{2}{3}} d x d t \\
& \quad \leq\left(\lambda_{1}+M\right)\left(\iint_{Q_{\omega}} u^{3} \varphi_{1} d x d t\right)^{\frac{1}{3}}\left(\iint_{Q_{\omega}} \varphi_{1} d x d t\right)^{\frac{2}{3}} \\
& \quad \leq\left(\lambda_{1}+M\right)\left(\iint_{Q_{\omega}} u^{3} \varphi_{1} d x d t\right)^{\frac{1}{3}}\left(\iint_{Q_{\omega}} c_{2} R(\Omega) d x d t\right)^{\frac{2}{3}} .
\end{aligned}
$$

By the above inequality we have

$$
\iint_{Q_{\omega}} u^{3} \varphi_{1}(x) d x d t \leq C .
$$

Using (4.19), we infer there exists a constant $C_{\varepsilon}>0$, such that

$$
\varphi_{1}(x) \geq C_{\varepsilon}, \quad x \in \Omega \backslash \Omega_{\varepsilon},
$$


where $\Omega_{\varepsilon}$ was defined in Lemma 4.2. Using (4.20) and (4.21), we deduce that

$$
\begin{aligned}
C_{\varepsilon} \int_{0}^{\omega} \int_{\Omega \backslash \Omega_{\varepsilon}} u^{3}(x, t) d x d t & \leq \int_{0}^{\omega} \int_{\Omega \backslash \Omega_{\varepsilon}} u^{3} \varphi_{1}(x) d x d t \\
& \leq \int_{0}^{\omega} \int_{\Omega} u^{3} \varphi_{1}(x) d x d t \\
& \leq C .
\end{aligned}
$$

Using Lemma 4.2 and (4.22), we obtain

$$
\iint_{Q_{\omega}} u^{3} d x d t \leq C
$$

Multiplying the first equation of (4.2) by $u_{t}$, integrating over $Q_{\omega}$, and using the periodicity of $u$ and (4.15), we obtain

$$
\begin{aligned}
& \|u\|_{W_{\omega}^{1,1 ; 2}\left(Q_{\omega}\right)}^{2} \\
& \quad=\iint_{Q_{\omega}}|\nabla u|^{2}+u_{t}^{2} d x d t \\
& \quad=\iint_{Q_{\omega}} m(t)\left(u^{4}-u^{2}+L u^{2}\right) d x d t-\iint_{Q_{\omega}} m^{\prime}(t)\left(\frac{u^{4}}{4}-\frac{u^{2}}{2}+\frac{L u^{2}}{2}\right) d x d t \\
& =\iint_{Q_{\omega}}\left(m(t)-\frac{m^{\prime}(t)}{4}\right) u^{4} d x d t+\iint_{Q_{\omega}}\left(m(t)-\frac{m^{\prime}(t)}{2}\right)(L-1) u^{2} d x d t .
\end{aligned}
$$

Using (4.24), we have

$$
\|u\|_{W_{\omega}^{1,1 ; 2}\left(Q_{\omega}\right)}^{2} \leq C\|u\|_{L^{4}\left(Q_{\omega}\right)}^{4} .
$$

By the interpolation inequality, using (4.23), we have

$$
\|u\|_{4}^{4} \leq\left(\|u\|_{3}^{\theta}\|u\|_{5}^{1-\theta}\right)^{4} \leq C\|u\|_{5}^{4(1-\theta)}
$$

where

$$
\frac{1}{4}=\frac{\theta}{3}+\frac{1-\theta}{5}, \quad \theta=\frac{3}{8}
$$

Using the Sobolev embedding theorem, we have

$$
\|u\|_{L^{5}\left(Q_{\omega}\right)}^{5} \leq C\|u\|_{W_{\omega}^{1,1 ; 2}\left(Q_{\omega}\right)}^{2}, \quad N=1,2 .
$$

Combining (4.25), (4.26), and (4.27), using Young's inequality with $\varepsilon$, we have

$$
\begin{aligned}
& \|u\|_{W_{\omega}^{1,1 ; 2}\left(Q_{\omega}\right)}^{2} \\
& \leq C\|u\|_{L^{4}\left(Q_{\omega}\right)}^{4} \\
& \leq C\|u\|_{5}^{4(1-\theta)} \\
& =C\left(\|u\|_{5}^{5}\right)^{\frac{4(1-\theta)}{5}}
\end{aligned}
$$




$$
\begin{aligned}
& \leq C\left(C\|u\|_{W_{\omega}^{1,1,2}\left(Q_{\omega}\right)}^{2}\right)^{\frac{4(1-\theta)}{5}} \\
& \leq \frac{\varepsilon\left(\left(\|u\|_{W_{\omega}^{1,1 ; 2}\left(Q_{\omega}\right)}^{2}\right)^{\frac{4(1-\theta)}{5}}\right)^{\eta}}{\eta}+\frac{\varepsilon^{\frac{1}{1-\eta}}\left(C C^{\frac{4(1-\theta)}{5}}\right)^{\frac{\eta}{\eta-1}}}{\frac{\eta}{\eta-1}} \\
& \leq \varepsilon\|u\|_{W_{\omega}^{1,1 ; 2}\left(Q_{\omega}\right)}^{2}+C,
\end{aligned}
$$

where $\eta:=\frac{5}{4(1-\theta)}>1$. Choosing a sufficiently small $\varepsilon$, by (4.28) we obtain

$$
\|u\|_{W_{\omega}^{11,12}\left(Q_{\omega}\right)}^{2} \leq C,
$$

where the constant $C$ is independent of $L$ and $u$. The proof of this lemma is complete.

Lemma 4.5 For $N=3$, suppose the assumptions $\left(\mathrm{H}_{1}\right)$ and $\left(\mathrm{H}_{2}\right)$ hold, if $u$ is a solution of the problem (4.2), then

$$
\|u\|_{W_{\omega}^{1,1 ; 2}\left(Q_{\omega}\right)}^{2}=\iint_{Q_{\omega}}|\nabla u|^{2}+u_{t}^{2} d x d t \leq C,
$$

where the positive constant $C$ is independent of $L$ and $u$.

Proof We calculate the first term on the right-hand side of (4.6). Integrating over $Q_{\omega}$, using the periodicity of $u$ and (4.9), we have

$$
\begin{aligned}
\iint_{Q_{\omega}} u_{t} \Delta u d x d t & =\int_{0}^{\omega} \int_{\partial \Omega}(\nabla u \cdot \vec{n}) u_{t} d s d t-\iint_{Q_{\omega}} \nabla u \cdot \nabla u_{t} d x d t \\
& =-\iint_{Q_{\omega}} \frac{d}{d t}\left(\frac{\nabla u \cdot \nabla u}{2}\right) d x d t \\
& =0
\end{aligned}
$$

where $d s$ is the surface area element of $\partial \Omega$. Recalling $R(\Omega)=\sup _{x \in \Omega}|x|$, thus $|x|^{2} \leq R^{2}(\Omega)$. Multiplying the first equation of the problem (4.2) by $u_{t}$, by (4.30), we obtain

$$
\begin{aligned}
\iint_{Q_{\omega}} u_{t}^{2} \frac{|x|^{2}}{2} d x d t & \leq \frac{R^{2}(\Omega)}{2} \iint_{Q_{\omega}} u_{t}^{2} d x d t \\
& =\frac{R^{2}(\Omega)}{2} \iint_{Q_{\omega}} m(t)\left(\left(u^{3}-u+L u\right) u_{t}\right) d x d t \\
& =\frac{R^{2}(\Omega)}{2} \iint_{Q_{\omega}} m(t) \frac{\partial\left(\frac{u^{4}}{4}-\frac{u^{2}}{2}+\frac{L u^{2}}{2}\right)}{\partial t} d x d t \\
& =-\frac{R^{2}(\Omega)}{2} \iint_{Q_{\omega}} m^{\prime}(t)\left(\frac{u^{4}}{4}-\frac{u^{2}}{2}+\frac{L u^{2}}{2}\right) d x d t .
\end{aligned}
$$

Substituting (4.31) into (4.6), we have

$$
\begin{aligned}
& \iint_{Q_{\omega}}\left(\left(1-\frac{N}{4}\right) u^{4}-u^{2}+L u^{2}\right) m(t) d x d t \\
& \quad+\iint_{Q_{\omega}}\left(\frac{R^{2}(\Omega)-|x|^{2}}{2}\left(\frac{u^{4}}{4}-\frac{u^{2}}{2}+\frac{L u^{2}}{2}\right) m^{\prime}(t)\right) d x d t
\end{aligned}
$$




$$
\begin{aligned}
= & \iint_{Q_{\omega}}\left\{\left(1-\frac{N}{4}\right) m(t)+\frac{R^{2}(\Omega)-|x|^{2}}{8} m^{\prime}(t)\right\} u^{4} d x d t \\
& +\iint_{Q_{\omega}}\left(m(t)+\frac{R^{2}(\Omega)-|x|^{2}}{4} m^{\prime}(t)\right)(L-1) u^{2} d x d t \\
\leq & \frac{1}{2} \iint_{\partial \Omega \times(0, \omega)}|\nabla u|^{2}(x \cdot \vec{n}) d s d t .
\end{aligned}
$$

Using Lemma 4.2 and (4.13), we have

$$
\begin{aligned}
\frac{1}{2} \iint_{\partial \Omega \times(0, \omega)}|\nabla u|^{2}(x \cdot \vec{n}) d s d t & \leq \frac{C}{2} \int_{0}^{\omega}\left(\int_{\partial \Omega}(x \cdot \vec{n}) d s\right) d t \\
& =\frac{C}{2} \int_{0}^{\omega}\left(\int_{\Omega} \operatorname{div} x d x\right) d t \\
& =\frac{C}{2} \int_{0}^{\omega} N|\Omega| d t \\
& \leq C .
\end{aligned}
$$

Recalling (4.24), we obtain

$$
\begin{aligned}
& \|u\|_{W_{\omega}^{1,1 ; 2}\left(Q_{\omega}\right)}^{2} \\
& \quad=\iint_{Q_{\omega}}\left(m(t)-\frac{m^{\prime}(t)}{4}\right) u^{4} d x d t+\iint_{Q_{\omega}}\left(m(t)-\frac{m^{\prime}(t)}{2}\right)(L-1) u^{2} d x d t .
\end{aligned}
$$

Using (4.32) and (4.34), by the assumption $\left(\mathrm{H}_{2}\right)$, we infer there is a constant $\widetilde{C}>0$ and a point $x \in \mathbb{R}^{N}$, such that

$$
m(t)-\frac{m^{\prime}(t)}{4} \leq \widetilde{C}\left\{\left(1-\frac{N}{4}\right) m(t)+\frac{R^{2}(\Omega)-|x|^{2}}{8} m^{\prime}(t)\right\}, \quad N=3 .
$$

In fact, if $m^{\prime}(t) \geq 0$, then we choose the constant $\widetilde{C} \geq 4$, thus (4.35) is valid. If $m^{\prime}(t)<0$, by the assumption $\left(\mathrm{H}_{1}\right)$, we have $0 \leq\left|m^{\prime}(t)\right| \leq C, 0<\left(m(t)-\frac{m^{\prime}(t)}{4}\right) \leq C$. In order to let

$$
\left\{\left(1-\frac{N}{4}\right) m(t)+\frac{R^{2}(\Omega)-|x|^{2}}{8} m^{\prime}(t)\right\}>0
$$

namely

$$
\sup _{t \in[0, \omega]} \frac{\left(m^{\prime}(t)\right)^{-}}{m(t)}<\frac{2(4-N)}{R^{2}(\Omega)-|x|^{2}}, \quad N=3,
$$

where $\left(m^{\prime}(t)\right)^{-}=\max \left\{0,-m^{\prime}(t)\right\}$, we choose $x$ as origin, $|x|^{2}=0$ in (4.36), thus (4.35) is valid since $m(t)$ satisfies the assumption $\left(\mathrm{H}_{2}\right)$. In addition, since $0 \leq L \leq L_{0}$, using (4.32), (4.33), (4.34), and (4.35), we get

$$
\|u\|_{W_{\omega}^{1,1,2}\left(Q_{\omega}\right)}^{2} \leq \frac{\widetilde{C}}{2} \iint_{\partial \Omega \times(0, \omega)}|\nabla u|^{2}(x \cdot \vec{n}) d s d t \leq C .
$$

The proof of this lemma is complete. 
Lemma 4.6 For $N \leq 3, L \in\left[0, L_{0}\right]$, if $u$ is a solution of the problem (4.2), then there exists a positive constant $C$, which is independent of $L$ and $u$, such that

$$
\|u\|_{L_{\omega}^{\infty}\left(Q_{\omega}\right)} \leq C
$$

Proof We proceed in a similar way to Proposition 17 in [13] (see also Proposition 2.2 in [15]). This proof is divided into three steps. In the first step, we prove the boundedness of $u$ in $L_{\omega}^{\infty}\left([0, \omega] ; L^{2}(\Omega)\right)$. In the second step, we will also need the following interpolation estimates (see (2.10) in [30]):

$$
W^{1,2}\left([0, \omega], L^{2}(\Omega)\right) \cap L^{2 q}\left([0, \omega], H^{1}(\Omega)\right) \hookrightarrow L^{\infty}\left([0, \omega], L^{\rho}(\Omega)\right),
$$

where $q \geq 1, \rho \in\left[2, \rho_{\max }\right)$, and

$$
\rho_{\max }= \begin{cases}{\left[2, \frac{2 N(q+1)}{q(N-2)+N}\right),} & N \geq 2, \\ \infty, & N=1 .\end{cases}
$$

We obtain the boundedness of $u$ in $L_{\omega}^{\infty}\left([0, \omega] ; L^{\rho}(\Omega)\right)$ for any $\rho \in\left[2, \rho_{\max }\right)$. In the third step, we based on interpolation and Morse-type iteration technique (see Theorem 16.4 in [29]) to prove $u$ is bounded in $L_{\omega}^{\infty}\left(Q_{\omega}\right)$.

First step: $u$ is bounded in $L_{\omega}^{\infty}\left([0, \omega] ; L^{2}(\Omega)\right)$.

By Lemma 4.4 and Lemma 4.5 , we are informed that there exists a $t_{0} \in[0, \omega]$, such that $\left\|u\left(t_{0}\right)\right\|_{L^{2}(\Omega)} \leq C$ and $\left\|u_{\tau}\right\|_{L^{2}\left(Q_{\omega}\right)} \leq C$. We notice that

$$
u(t)=u\left(t_{0}\right)+\int_{t_{0}}^{t} u_{\tau} d \tau
$$

Then

$$
(u(t))^{2} \leq 2\left(u\left(t_{0}\right)\right)^{2}+2\left(\int_{t_{0}}^{t} u_{\tau} d \tau\right)^{2} \leq 2\left(u\left(t_{0}\right)\right)^{2}+2 \int_{t_{0}}^{t}\left(u_{\tau}\right)^{2} d \tau \int_{t_{0}}^{t} d \tau .
$$

From the above equality, we have

$$
\begin{aligned}
\|u\|_{L_{\omega}^{\infty}\left([0, \omega] ; L^{2}(\Omega)\right)} & =\sup _{t \in[0, \omega]}\left(\int_{\Omega}|u(t)|^{2} d x\right)^{\frac{1}{2}} \\
& \leq \sup _{t \in[0, \omega]}\left(2 \int_{\Omega}\left|u\left(t_{0}\right)\right|^{2} d x+2 \omega \int_{\Omega} \int_{t_{0}}^{t}\left|u_{\tau}\right|^{2} d \tau d x\right)^{\frac{1}{2}} \\
& \leq C .
\end{aligned}
$$

Second step: $u$ is bounded in $L_{\omega}^{\infty}\left([0, \omega] ; L^{\rho}(\Omega)\right)$ for any $\rho \in\left[2, \rho_{\max }\right)$.

For $t \in[0, \omega]$, we define the 'action' function as follows:

$$
S(u(t))=\frac{1}{2} \int_{\Omega}|\nabla u(t)|^{2} d x-\int_{\Omega} m(t)\left(\frac{u^{4}}{4}-\frac{u^{2}}{2}+\frac{L u^{2}}{2}\right) d x, \quad L \in\left[0, L_{0}\right] .
$$


We first prove that $S(u(\cdot))$ is bounded in $L_{\omega}^{1}(0, \omega)$. From the equality (4.39), Lemma 4.4, and Lemma 4.5, we find

$$
\begin{aligned}
& \|S(u(\cdot))\|_{L^{1}(0, \omega)} \\
& =\int_{0}^{\omega}|S(u(t))| d t \\
& \leq \frac{1}{2} \iint_{Q_{\omega}}|\nabla u(t)|^{2} d x d t+\iint_{Q_{\omega}}\left|m(t)\left(\frac{u^{4}}{4}-\frac{u^{2}}{2}+\frac{L u^{2}}{2}\right)\right| d x d t \\
& =\frac{1}{2} \iint_{Q_{\omega}} m(t)\left(u^{4}-u^{2}+L u^{2}\right) d x d t \\
& \quad+\iint_{Q_{\omega}}\left|m(t)\left(\frac{u^{4}}{4}-\frac{u^{2}}{2}+\frac{L u^{2}}{2}\right)\right| d x d t \\
& \leq C \iint_{Q_{\omega}} m(t) u^{4} d x d t \\
& \leq C .
\end{aligned}
$$

Therefore, $S(u(\cdot))$ is bounded in $L_{\omega}^{1}(0, \omega)$. Moreover, for any $t, s \in[0, \omega]$, from (4.39) we have

$$
\begin{aligned}
S(u(t))-S(u(s)) \\
=\int_{s}^{t} \frac{\partial S(u(\tau))}{\partial \tau} d \tau \\
=\frac{1}{2} \int_{s}^{t} \frac{\partial}{\partial \tau}\left(\int_{\Omega}|\nabla u(\tau)|^{2} d x\right) d \tau-\int_{s}^{t} \int_{\Omega} m^{\prime}(\tau)\left(\frac{u^{4}}{4}-\frac{u^{2}}{2}+\frac{L u^{2}}{2}\right) d x d \tau \\
\quad-\int_{s}^{t} \int_{\Omega} m(\tau)\left(u^{3}-u+L u\right) \frac{\partial u}{\partial \tau} d x d \tau .
\end{aligned}
$$

Using the first equation of the problem (4.2), we infer

$$
\begin{aligned}
& \int_{s}^{t} \int_{\Omega} m(\tau)\left(u^{3}-u+L u\right) \frac{\partial u}{\partial \tau} d x d \tau \\
& \quad=\int_{s}^{t} \int_{\Omega}\left(\frac{\partial u}{\partial \tau}-\Delta u\right) \frac{\partial u}{\partial \tau} d x d \tau \\
& =\int_{s}^{t} \int_{\Omega}\left|\frac{\partial u}{\partial \tau}\right|^{2} d x d \tau+\int_{s}^{t} \int_{\Omega} \nabla u \cdot \nabla u_{\tau} d x d \tau \\
& =\int_{s}^{t} \int_{\Omega}\left|\frac{\partial u}{\partial \tau}\right|^{2} d x d \tau+\frac{1}{2} \int_{s}^{t} \frac{\partial}{\partial \tau}\left(\int_{\Omega}|\nabla u(\tau)|^{2} d x\right) d \tau .
\end{aligned}
$$

The above inequality implies

$$
\begin{aligned}
& \frac{1}{2} \int_{s}^{t} \frac{\partial}{\partial \tau}\left(\int_{\Omega}|\nabla u(\tau)|^{2} d x\right) d \tau-\int_{s}^{t} \int_{\Omega} m(\tau)\left(u^{3}-u+L u\right) \frac{\partial u}{\partial \tau} d x d \tau \\
& \quad=-\int_{s}^{t} \int_{\Omega}\left|\frac{\partial u}{\partial \tau}\right|^{2} d x d \tau .
\end{aligned}
$$


Lemma 4.4 and Lemma 4.5 imply

$$
\begin{aligned}
& \int_{s}^{t} \int_{\Omega}\left|m^{\prime}(\tau)\left(\frac{u^{4}}{4}-\frac{u^{2}}{2}+\frac{L u^{2}}{2}\right)\right| d x d \tau \leq C, \\
& \int_{s}^{t} \int_{\Omega}\left|\frac{\partial u}{\partial \tau}\right|^{2} d x d \tau \leq\|u\|_{W^{1,1 ; 2}\left(Q_{\omega}\right)}^{2} \leq C .
\end{aligned}
$$

Substituting (4.42) into (4.41), we use (4.43) to get, for $s, t \in[0, \omega]$,

$$
\begin{aligned}
& |S(u(t))-S(u(s))| \\
& \quad=\left.\left|-\int_{s}^{t} \int_{\Omega} m^{\prime}(\tau)\left(\frac{u^{4}}{4}-\frac{u^{2}}{2}+\frac{L u^{2}}{2}\right) d x d \tau-\int_{s}^{t} \int_{\Omega}\right| \frac{\partial u}{\partial \tau}\right|^{2} d x d \tau \mid \\
& \quad \leq \int_{s}^{t} \int_{\Omega}\left|m^{\prime}(\tau)\left(\frac{u^{4}}{4}-\frac{u^{2}}{2}+\frac{L u^{2}}{2}\right)\right| d x d \tau+\int_{s}^{t} \int_{\Omega}\left|\frac{\partial u}{\partial \tau}\right|^{2} d x d \tau \\
& \quad \leq C .
\end{aligned}
$$

From (4.40) we infer that there exists a point $s \in[0, \omega]$ such that

$$
S(u(s))(\omega-0)=\int_{0}^{\omega}|S(u(t))| d t \leq C .
$$

Combining (4.44) and (4.45), we get

$$
\sup _{t \in[0, \omega]}|S(u(t))| \leq \sup _{t \in[0, \omega]}(|S(u(t))-S(u(s))|+|S(u(s))|) \leq C .
$$

We multiply the first equation of the problem (4.2) by $u$ and integrate over $\Omega$, then

$$
\int_{\Omega} u u_{t} d x+\int_{\Omega}|\nabla u(t)|^{2} d x=\int_{\Omega} m(t)\left(u^{4}-u^{2}+L u^{2}\right) d x
$$

Substituting (4.39) into (4.47), we get

$$
\begin{aligned}
\int_{\Omega}|\nabla u|^{2} d x= & \int_{\Omega} u u_{t} d x+4 S(u(t)) \\
& -\int_{\Omega} m(t) u^{2} d x+L \int_{\Omega} m(t) u^{2} d x
\end{aligned}
$$

From (4.38) we have

$$
\sup _{t \in[0, \omega]} \int_{\Omega} u^{2} d x \leq C
$$

By (4.46), (4.48), and (4.49), we infer

$$
\int_{\Omega}|\nabla u|^{2} d x \leq \int_{\Omega}\left|u u_{t}\right| d x+C .
$$


Using (4.49) and (4.43), we infer from (4.50) that

$$
\begin{aligned}
\int_{s}^{t}\left(\int_{\Omega}|\nabla u|^{2} d x\right)^{2} d \tau & \leq \int_{s}^{t}\left(\int_{\Omega}\left|u u_{\tau}\right| d x+C\right)^{2} d \tau \\
& \leq C \int_{s}^{t}\left(\int_{\Omega}\left|u u_{\tau}\right| d x\right)^{2} d \tau+C \\
& \leq C \int_{s}^{t}\left(\int_{\Omega} u^{2} d x\right)\left(\int_{\Omega} u_{\tau}^{2} d x\right) d \tau+C \\
& \leq C \int_{s}^{t} \int_{\Omega} u_{\tau}^{2} d x d \tau+C \\
& \leq C
\end{aligned}
$$

Hence we infer from (4.51) that

$$
\|u\|_{L^{4}\left([0, \omega], H^{1}(\Omega)\right)} \leq C
$$

For $N \geq 4$, if $\Omega$ is star shaped, then there is no nontrivial periodic solution for the problem (1.1)-(1.3) (see Section 6 for the proof of Theorem 2.2). Thus we consider the case of $N \leq 3$. For $N=3$, we will obtain the $L_{\omega}^{\infty}\left(Q_{\omega}\right)$ estimates, which will also be valid for $N=1$, 2. Lemma 4.5 implies $u \in W^{1,2}\left([0, \omega], L^{2}(\Omega)\right)$. In fact, using Lemma 4.5 and Hölder's inequality, we have

$$
\begin{aligned}
\|u\|_{W^{1,2}\left([0, \omega], L^{2}(\Omega)\right)}^{2} & =\int_{0}^{\omega}\left|\frac{\partial}{\partial t}\left(\int_{\Omega} u^{2} d x\right)^{\frac{1}{2}}\right|^{2} d t \\
& =\int_{0}^{\omega}\left|\left(\int_{\Omega} u^{2} d x\right)^{\frac{-1}{2}} \int_{\Omega} u u_{t} d x\right|^{2} d t \\
& \leq \int_{0}^{\omega}\left|\left(\int_{\Omega} u^{2} d x\right)^{\frac{-1}{2}}\left(\int_{\Omega} u^{2} d x\right)^{\frac{1}{2}}\left(\int_{\Omega}\left|u_{t}\right|^{2} d x\right)^{\frac{1}{2}}\right|^{2} d t \\
& =\int_{0}^{\omega} \int_{\Omega}\left|u_{t}\right|^{2} d x d t \\
& \leq\|u\|_{W^{1,1 ; 2}\left(Q_{\omega}\right)}^{2} \\
& \leq C .
\end{aligned}
$$

For the case of $N=3, q=2$, using (4.37), (4.52), and (4.53), we have

$$
\begin{aligned}
& W^{1,2}\left([0, \omega], L^{2}(\Omega)\right) \cap L^{4}\left([0, \omega], H^{1}(\Omega)\right) \\
& \quad \hookrightarrow L^{\infty}\left([0, \omega], L^{\rho}(\Omega)\right), \quad N=3, \rho \in\left[2, \frac{18}{5}\right) .
\end{aligned}
$$

Thus

$$
\sup _{t \in[0, \omega]} \int_{\Omega}|u|^{\frac{7}{2}} d x \leq C .
$$


We replace $q=2$ with $q=\frac{7}{2}$, repeating the process of (4.50)-(4.51), to obtain

$$
\begin{aligned}
\int_{s}^{t}\left(\int_{\Omega}|\nabla u|^{2} d x\right)^{\frac{7}{2}} d \tau & \leq \int_{s}^{t}\left(\int_{\Omega}\left|u u_{\tau}\right| d x+C\right)^{\frac{7}{2}} d \tau \\
& \leq C \int_{s}^{t}\left(\int_{\Omega}\left|u u_{\tau}\right| d x\right)^{\frac{7}{2}} d \tau+C \\
& \leq C \int_{s}^{t}\left(\int_{\Omega}|u|^{\frac{7}{2}} d x\right)\left(\int_{\Omega}\left|u_{\tau}\right|^{\frac{7}{5}} d x\right)^{\frac{5}{2}} d \tau+C \\
& \leq C \int_{s}^{t}\left(\int_{\Omega}\left|u_{\tau}\right|^{\frac{7}{5}} d x\right)^{\frac{5}{2}} d \tau+C \\
& \leq C \int_{s}^{t}\left(\int_{\Omega} 1 d x\right)^{\frac{3}{4}}\left(\int_{\Omega}\left|u_{\tau}\right|^{2} d x\right)^{\frac{7}{4}} d \tau+C \\
& \leq C .
\end{aligned}
$$

Hence we infer from (4.54) that

$$
u \text { is bounded in } L^{7}\left([0, \omega], H^{1}(\Omega)\right) .
$$

For the case of $N=3, q=\frac{7}{2}$, using (4.37), (4.53) and (4.55), we have

$$
\begin{gathered}
W^{1,2}\left([0, \omega], L^{2}(\Omega)\right) \cap L^{7}\left([0, \omega], H^{1}(\Omega)\right) \\
\hookrightarrow L^{\infty}\left([0, \omega], L^{\rho}(\Omega)\right), \quad \rho \in\left[2, \frac{54}{13}\right) .
\end{gathered}
$$

Third step: $u$ is bounded in $L_{\omega}^{\infty}\left(Q_{\omega}\right)$.

We based on interpolation and Morse-type iteration technique (see Theorem 16.4 in [29]) to prove $u$ is bounded in $L_{\omega}^{\infty}\left(Q_{\omega}\right)$. For $r \geq 4$, multiplying (1.1) by $u^{2 r-1}$ and integrate over $\Omega$, we obtain, for any $L \in\left[0, L_{0}\right]$,

$$
\frac{1}{2 r} \frac{d}{d t} \int_{\Omega} u^{2 r} d x+\frac{2 r-1}{r^{2}} \int_{\Omega}\left|\nabla\left(u^{r}\right)\right|^{2} d x=\int_{\Omega} m(t)\left(u^{2+2 r}-u^{2 r}+L u^{2 r}\right) d x .
$$

In this step, we denote $\|\cdot\|_{p}$ the $L^{p}(\Omega)$ norms, and

$$
w:=u^{r}, \quad \alpha(r):=\frac{1+r}{r} .
$$

By interpolation inequality for $L^{2 \alpha}(\Omega)$ norms, we have

$$
\|w\|_{2 \alpha}^{2 \alpha} \leq\left(\|w\|_{1}^{\beta}\|w\|_{2^{*}}^{1-\beta}\right)^{2 \alpha}
$$

where $1 \leq 2 \alpha \leq 2^{*}:=\frac{2 N}{N-2}$, and

$$
\frac{1}{2 \alpha}=\frac{\beta}{1}+\frac{1-\beta}{2^{*}}, \quad \beta(r) \in(0,1)
$$


For $N=3,2^{*}=6$, from the above equality, we have

$$
\beta(r)=\frac{2 r-1}{5(r+1)}
$$

Using the identity (4.57), interpolation inequality (4.59), the Sobolev embedding theorem, and Young's inequality, we obtain

$$
\begin{aligned}
\frac{1}{2 r} \frac{d}{d t}\|w\|_{2}^{2}+\frac{2 r-1}{r^{2}}\|\nabla w\|_{2}^{2} & \leq\|m(t)\|_{L^{\infty}[0, \omega]}\left(\|w\|_{2 \alpha}^{2 \alpha}+L\|w\|_{2}^{2}\right) \\
& \leq C\|w\|_{2 \alpha}^{2 \alpha} \\
& \leq C\left(\|w\|_{1}^{\beta}\|w\|_{2^{*}}^{1-\beta}\right)^{2 \alpha} \\
& \leq C\left(\|w\|_{1}^{\beta}\|\nabla w\|_{2}^{1-\beta}\right)^{2 \alpha} \\
& \leq\left(\frac{1}{r}\|\nabla w\|_{2}^{2}\right)^{\alpha(1-\beta)} C\left(r^{1-\beta}\|w\|_{1}^{2 \beta}\right)^{\alpha} \\
& \leq \frac{\left(\left(\frac{1}{r}\|\nabla w\|_{2}^{2}\right)^{\alpha(1-\beta)}\right)^{\frac{1}{1-\gamma}}+\frac{\left(C\left(r^{1-\beta}\|w\|_{1}^{2 \beta}\right)^{\alpha}\right)^{\frac{1}{\gamma}}}{\frac{1}{1-\gamma}}}{} \\
& \leq \frac{1}{r}\|\nabla w\|_{2}^{2}+C^{\frac{1}{\gamma}} r^{\frac{\alpha(1-\beta)}{\gamma}}\|w\|_{1}^{\frac{2 \alpha \beta}{\gamma}}
\end{aligned}
$$

where

$$
\gamma:=1-\alpha(1-\beta)=\frac{2 r-6}{5 r}
$$

Since we will choose the parameter $r \geq 4,0<\gamma<1$. Moving the term $\frac{1}{r}\|\nabla w\|_{2}^{2}$ of (4.61) to the left side, multiplying (4.61) with $r$, we obtain

$$
\frac{1}{2} \frac{d}{d t}\|w\|_{2}^{2}+\frac{r-1}{r}\|\nabla w\|_{2}^{2} \leq C^{\frac{1}{\gamma}} r^{1+\frac{\alpha(1-\beta)}{\gamma}}\|w\|_{1}^{\frac{2 \alpha \beta}{\gamma}} .
$$

Integrating (4.63) over $[\tau, \tau+\omega]$, using the periodicity of $u$, we get

$$
\begin{aligned}
\frac{r-1}{r} \int_{\tau}^{\tau+\omega}\|\nabla w\|_{2}^{2} d t & \leq \int_{\tau}^{\tau+\omega} C^{\frac{1}{\gamma}} r^{1+\frac{\alpha(1-\beta)}{\gamma}} \sup _{t \in[\tau, \tau+\omega]}\|w\|_{1}^{\frac{2 \alpha \beta}{\gamma}} d t \\
& =\omega C^{\frac{1}{\gamma}} r^{1+\frac{\alpha(1-\beta)}{\gamma}} \sup _{t \in[\tau, \tau+\omega]}\|w\|_{1}^{\frac{2 \alpha \beta}{\gamma}} .
\end{aligned}
$$

Using the Poincaré inequality, we see that there exists a constant $C>0$, such that

$$
\|w\|_{2}^{2} \leq C\|\nabla w\|_{2}^{2}
$$

Consequently, from (4.64) and (4.65), we infer

$$
\int_{\tau}^{\tau+\omega}\|u\|_{2 r}^{2 r} d t \leq \omega C^{\frac{1}{\gamma}} r^{1+\frac{\alpha(1-\beta)}{\gamma}} \sup _{t \in[\tau, \tau+\omega]}\|w\|_{1}^{\frac{2 \alpha \beta}{\gamma}}
$$


By the integral mean value theorem, using (4.66), we infer that there exists a point $\hat{t} \in$ $(\tau, \tau+\omega)$, such that

$$
\int_{\Omega}|u(x, \hat{t})|^{2 r} d x \leq C^{\frac{1}{\gamma}} r^{1+\frac{\alpha(1-\beta)}{\gamma}} \sup _{t \in[\tau, \tau+\omega]}\|w\|_{1}^{\frac{2 \alpha \beta}{\gamma}}
$$

Integrating (4.63) over $[\hat{t}, t]$, we have

$$
\begin{aligned}
& \frac{1}{2} \int_{\Omega}|u(x, t)|^{2 r} d x-\frac{1}{2} \int_{\Omega}|u(x, \hat{t})|^{2 r} d x+\frac{r-1}{r} \int_{\hat{t}}^{t}\|\nabla w\|_{2}^{2} d t \\
& \quad \leq(t-\hat{t}) C^{\frac{1}{\gamma}} r^{1+\frac{\alpha(1-\beta)}{\gamma}} \sup _{t \in[\tau, \tau+\omega]}\|w\|_{1}^{\frac{2 \alpha \beta}{\gamma}}, \quad t \in[\hat{t}, \tau+\omega] .
\end{aligned}
$$

Combining the above inequality with (4.64) and (4.67), we obtain

$$
\int_{\Omega}|u(x, t)|^{2 r} d x \leq C^{\frac{1}{\gamma}} r^{1+\frac{\alpha(1-\beta)}{\gamma}} \sup _{t \in[\tau, \tau+\omega]}\|w\|_{1}^{\frac{2 \alpha \beta}{\gamma}}, \quad t \in[\hat{t}, \tau+\omega] .
$$

Taking the $2 r$ th root of both sides of the above inequality, we have

$$
\|u\|_{2 r}=\left(\int_{\Omega} u^{2 r} d x\right)^{\frac{1}{2 r}} \leq C^{\frac{1}{2 r \gamma}} r^{\left(1+\frac{\alpha(1-\beta)}{\gamma}\right) \frac{1}{2 r}} \sup _{t \in[\tau, \tau+\omega]}\|w\|_{1}^{\frac{\alpha \beta}{r \gamma}}, \quad t \in[\hat{t}, \tau+\omega] .
$$

Using (4.58) and (4.60), recalling the definition of $\gamma$ in (4.62), we define two functions $\delta(r), \rho(r)$ as follows:

$$
\begin{aligned}
& \delta(r):=\frac{1}{2 r \gamma}=\frac{5}{4 r-12}, \\
& \left(1+\frac{\alpha(1-\beta)}{\gamma}\right) \frac{1}{2 r}=\frac{5}{4 r-12}=\delta(r), \\
& \rho(r):=\frac{\alpha \beta}{r \gamma}=\frac{2 r-1}{r(2 r-6)} .
\end{aligned}
$$

Noticing the periodicity of $u$, choosing $t=\tau+\omega$ in (4.68), combining with (4.69), we obtain

$$
\begin{aligned}
\|u(x, \tau)\|_{2 r} & =\|u(x, \tau+\omega)\|_{2 r} \leq C^{\frac{1}{2 r \gamma}} r^{\left(1+\frac{\alpha(1-\beta)}{\gamma}\right) \frac{1}{2 r}} \sup _{t \in[\tau, \tau+\omega]}\|w\|_{1}^{\frac{\alpha \beta}{\gamma \gamma}} \\
& =C^{\delta(r)} r^{\delta(r)} \sup _{t \in[\tau, \tau+\omega]}\|u\|_{r}^{\rho(r)} .
\end{aligned}
$$

Integrating (4.63) over $[\tau, t]$, we have

$$
\begin{aligned}
& \frac{1}{2} \int_{\Omega}|u(x, t)|^{2 r} d x-\frac{1}{2} \int_{\Omega}|u(x, \tau)|^{2 r} d x+\frac{r-1}{r} \int_{t}^{\tau}\|\nabla w\|_{2}^{2} d t \\
& \quad \leq \int_{\tau}^{t} C^{\frac{1}{\gamma}} r^{1+\frac{\alpha(1-\beta)}{\gamma}} \sup _{t \in[\tau, \tau+\omega]}\|w\|_{1}^{\frac{2 \alpha \beta}{\gamma}} d t \\
& \quad \leq(t-\tau) C^{\frac{1}{\gamma}} r^{2 r \delta(r)}\left(\sup _{t \in[\tau, \tau+\omega]}\|u\|_{r}\right)^{2 r \rho(r)}, \quad t \in[\tau, \hat{t}] .
\end{aligned}
$$


From (4.64), (4.70), and (4.71), we have

$$
\|u\|_{2 r}=\left(\int_{\Omega} u^{2 r} d x\right)^{\frac{1}{2 r}} \leq C^{\delta(r)} r^{\delta(r)} \sup _{t \in[\tau, \tau+\omega]}\|u\|_{r}^{\rho(r)}, \quad t \in[\tau, \hat{t}] .
$$

Using (4.68) and (4.72), for any $t \in[\tau, \tau+\omega]$, we have

$$
\sup _{t \in[\tau, \tau+\omega]}\|u\|_{2 r}=\sup _{t \in[\tau, \tau+\omega]}\left(\int_{\Omega} u^{2 r} d x\right)^{\frac{1}{2 r}} \leq C^{\delta(r)} r^{\delta(r)} \sup _{t \in[\tau, \tau+\omega]}\|u\|_{r}^{\rho(r)} .
$$

We define a constant $U_{r}$ as follows:

$$
U_{r}:=\sup _{t \in[\tau, \tau+\omega]}\|u\|_{r}=\sup _{t \in[\tau, \tau+\omega]}\left(\int_{\Omega} u^{r} d x\right)^{\frac{1}{r}}, \quad r \geq 4 .
$$

From (4.73), we have

$$
U_{2 r} \leq C^{\delta(r)} r^{\delta(r)} U_{r}^{\rho(r)}
$$

By a bootstrap argument, using (4.57) and replacing $r$ with $2^{k} r, k:=1,2,3, \ldots$, repeating the process of (4.57)-(4.73), we obtain

$$
U_{2^{k+1} r} \leq C^{\delta\left(2^{k} r\right)}\left(2^{k} r\right)^{\delta\left(2^{k} r\right)}\left(U_{2^{k}}\right)^{\rho\left(2^{k} r\right)} .
$$

In the following, we will fix the parameter $r=4$. Using (4.76), by iteration, we have

$$
\begin{aligned}
& U_{4 \times 2^{k+1}} \leq C^{\delta\left(4 \times 2^{k}\right)}\left(4 \times 2^{k}\right)^{\delta\left(4 \times 2^{k}\right)}\left(U_{4 \times 2^{k}}\right)^{\rho\left(4 \times 2^{k}\right)} \\
& \leq C^{\delta\left(4 \times 2^{k}\right)}\left(4 \times 2^{k}\right)^{\delta\left(4 \times 2^{k}\right)} \\
& \times\left(C^{\delta\left(4 \times 2^{k-1}\right)}\left(4 \times 2^{k-1}\right)^{\delta\left(4 \times 2^{k-1}\right)}\left(U_{4 \times 2^{k-1}}\right)^{\rho\left(4 \times 2^{k-1}\right)}\right)^{\rho\left(4 \times 2^{k}\right)} \\
& \leq C^{\delta\left(4 \times 2^{k}\right)}\left(4 \times 2^{k}\right)^{\delta\left(4 \times 2^{k}\right)}\left(C^{\delta\left(4 \times 2^{k-1}\right)}\left(4 \times 2^{k-1}\right)^{\delta\left(4 \times 2^{k-1}\right)}\right.
\end{aligned}
$$

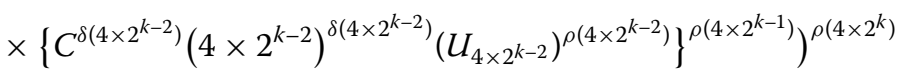

$$
\begin{aligned}
& \leq \cdots \\
& \leq(4 C)^{k_{1}} 2^{k_{2}}\left(U_{4}\right)^{k_{3}}
\end{aligned}
$$

where

$$
\begin{aligned}
k_{1}= & k_{1}(k) \\
= & \delta\left(4 \times 2^{k}\right)+\delta\left(4 \times 2^{k-1}\right) \rho\left(4 \times 2^{k}\right) \\
& +\delta\left(4 \times 2^{k-2}\right) \rho\left(4 \times 2^{k-1}\right) \rho\left(4 \times 2^{k}\right) \\
& +\cdots+\left(\delta\left(4 \times 2^{0}\right) \rho\left(4 \times 2^{1}\right) \rho\left(4 \times 2^{2}\right) \cdots \rho\left(4 \times 2^{k}\right)\right), \\
k_{2}= & k_{2}(k)
\end{aligned}
$$




$$
\begin{aligned}
= & k \delta\left(4 \times 2^{k}\right)+(k-1) \delta\left(4 \times 2^{k-1}\right) \rho\left(4 \times 2^{k}\right) \\
& +(k-2) \delta\left(4 \times 2^{(k-2)}\right) \rho\left(4 \times 2^{(k-1)}\right) \rho\left(4 \times 2^{k}\right) \\
& +\cdots+\left(1 \delta\left(4 \times 2^{1}\right) \rho\left(4 \times 2^{2}\right) \rho\left(4 \times 2^{3}\right) \cdots \rho\left(4 \times 2^{k}\right)\right), \\
k_{3}= & k_{3}(k)=\rho\left(4 \times 2^{k}\right) \rho\left(4 \times 2^{k-1}\right) \cdots \rho\left(4 \times 2^{2}\right) \rho\left(4 \times 2^{1}\right) \rho\left(4 \times 2^{0}\right) .
\end{aligned}
$$

Recalling the definition of $\rho(r)$ in (4.69), we obtain

$$
\begin{aligned}
& 0<\rho\left(4 \times 2^{k}\right) \leq \frac{7}{8}, \quad k=0,1,2, \ldots, \\
& 0<k_{3}(k)<1, \\
& \lim _{k \rightarrow+\infty} k_{3}(k)=0 .
\end{aligned}
$$

Obviously, $4 \times\left(4 \times 2^{i}\right)-12>2^{i}, i=0,1,2, \ldots$ Recalling the definition of $\delta(r)$ in (4.69), using (4.78) and (4.79), we have

$$
\begin{aligned}
& k_{1}(k)<<\left(4 \times 2^{k}\right)+\delta\left(4 \times 2^{k-1}\right)+\delta\left(4 \times 2^{k-2}\right)+\cdots+\delta\left(4 \times 2^{0}\right) \\
&= \sum_{i=0}^{k} \frac{5}{4 \times\left(4 \times 2^{i}\right)-12}<\sum_{i=0}^{k} \frac{5}{2^{i}}=10\left(1-\left(\frac{1}{2}\right)^{k+1}\right), \\
& \lim _{k \rightarrow+\infty} k_{1}(k)=10 .
\end{aligned}
$$

We also have

$$
\begin{aligned}
& k_{2}(k)< k \delta\left(4 \times 2^{k}\right)+(k-1) \delta\left(4 \times 2^{k-1}\right) \\
&+(k-2) \delta\left(4 \times 2^{k-2}\right)+\cdots+1 \delta\left(4 \times 2^{1}\right) \\
&= \sum_{i=0}^{k} \frac{5 i}{4 \times\left(4 \times 2^{i}\right)-12}<\sum_{i=1}^{k} \frac{5 i}{2^{i}}=10\left(1-\left(\frac{1}{2}\right)^{k}\right)-\frac{k}{2^{k}}, \\
& \lim _{k \rightarrow+\infty} k_{2}(k)=10 .
\end{aligned}
$$

Using (4.56), we have

$$
U_{4}=\sup _{t \in[\tau, \tau+\omega]}\|u\|_{4} \leq C
$$

From (4.77), (4.79), (4.80), (4.81), and (4.82), we obtain

$$
\|u\|_{L_{\omega}^{\infty}\left(Q_{\omega}\right)}=\lim _{k \rightarrow+\infty} U_{4 \times 2^{k+1}} \leq \lim _{k \rightarrow+\infty}(4 C)^{k_{1}} 2^{k_{2}}\left(U_{4}\right)^{k_{3}} \leq C,
$$

where the positive constant $C$ is independent of $k, u, L$. Thus, all solutions of the problem (4.2) are bounded in $L_{\omega}^{\infty}\left(Q_{\omega}\right)$.

Proposition $4.1 \operatorname{deg}\left(I-K_{1}, B_{R}, 0\right)=0$. 
Proof In fact, if $u$ is a solution of the problem (4.2), then Lemma 4.6 implies that $u$ is bounded in $L_{\omega}^{\infty}\left(Q_{\omega}\right)$, thus there exists a constant $R>\min \left\{r, C\left(L_{0}\right)\right\}$, where $r$ is defined as (3.6), $R$ is independent of $L, u$, such that

$$
\|u\|_{L_{\omega}^{\infty}\left(Q_{\omega}\right)} \leq C\left(L_{0}\right)<R, \quad L \in\left[0, L_{0}\right]
$$

where $L_{0}$ is defined as (4.1). From (4.83) we infer $T_{L}$ has no fixed point for $u \in \partial B_{R}$, namely

$$
T_{L} u \neq u, \quad L \in\left[0, L_{0}\right], \quad\|u\|_{L_{\omega}^{\infty}\left(Q_{\omega}\right)}=R .
$$

For $L=L_{0}$, Proposition 4.1 implies the problem (4.2) has no solutions, thus

$$
\operatorname{deg}\left(I-T_{L_{0}}, B_{R}, 0\right)=0
$$

Using the homotopy invariance of the degree, from (4.84) we infer

$$
\operatorname{deg}\left(I-T_{0}, B_{R}, 0\right)=\operatorname{deg}\left(I-T_{L_{0}}, B_{R}, 0\right)=0 .
$$

We notice that operator $K_{1}=T_{0}$, then

$$
\operatorname{deg}\left(I-K_{1}, B_{R}, 0\right)=\operatorname{deg}\left(I-T_{0}, B_{R}, 0\right)=0 .
$$

The proof of this proposition is complete.

\section{Annulus domains}

In this section, we consider the problem (1.1)-(1.3) in annulus domain $\Omega=B_{R_{0}} \backslash B_{r_{0}}$. In order to calculate $\operatorname{deg}\left(I-T_{l}, B_{\widehat{R}}, 0\right)$, we will obtain the $L_{\omega}^{\infty}\left(\left[s_{0}, s_{1}\right] \times[0, \omega]\right)$ estimates for radial solutions.

Let $r^{2}=|x|^{2}=x_{1}^{2}+x_{2}^{2}+\cdots+x_{N}^{2}$, where $N \geq 3$. Let $u(r, t)=u(|x|, t)=u(x, t)$ and $v(s, t)=$ $u(r, t)$ with $s=r^{-\beta}, \beta=N-2$. We have

$$
\begin{aligned}
\frac{\partial u}{\partial r} & =\frac{\partial v}{\partial s} \frac{\partial s}{\partial r}=-\beta r^{-\beta-1} \frac{\partial v}{\partial s}, \\
\Delta u & =\frac{\partial^{2} u}{\partial r^{2}}+\frac{N-1}{r} \frac{\partial u}{\partial r} \\
& =\beta^{2} r^{-2 \beta-2} \frac{\partial^{2} v}{\partial s^{2}}+\underbrace{\beta(\beta+1) r^{-\beta-2} \frac{\partial v}{\partial s}+\frac{N-1}{r}\left(-\beta r^{-\beta-1} \frac{\partial v}{\partial s}\right)}_{0} \\
& =\beta^{2} s^{\frac{2(N-1)}{N-2}} \frac{\partial^{2} v}{\partial s^{2}} .
\end{aligned}
$$

Then the problem (1.1)-(1.3) becomes

$$
\left\{\begin{array}{l}
\frac{1}{\beta^{2}} s^{-\frac{2(N-1)}{N-2}} \frac{\partial v}{\partial t}-v_{s s}=\frac{m(t)}{\beta^{2}} s^{-\frac{2(N-1)}{N-2}}\left(v^{3}-v\right), \quad(s, t) \in\left(s_{0}, s_{1}\right) \times \mathbb{R}^{+}, \\
v\left(s_{0}, t\right)=0, \quad v\left(s_{1}, t\right)=0, \\
v(s, t)=v(s, t+\omega)=0,
\end{array}\right.
$$

where $s_{0}=R_{0}^{-\beta}, s_{1}=r_{0}^{-\beta}, 0<s_{0}<s<s_{1}$. 
In order to employ the topological degree method to deal with the existence of the nontrivial periodic solutions of the problem (5.1), we introduce the operators $K_{\sigma}$ from $L_{\omega}^{\infty}\left(\left[s_{0}, s_{1}\right] \times[0, \omega]\right)$ to itself,

$$
\begin{aligned}
& K_{\sigma}: L_{\omega}^{\infty}\left(\left[s_{0}, s_{1}\right] \times[0, \omega]\right) \times[0,1] \rightarrow L_{\omega}^{\infty}\left(\left[s_{0}, s_{1}\right] \times[0, \omega]\right), \\
& (u, \sigma) \mapsto v .
\end{aligned}
$$

We say that $K_{\sigma} u=v$, if $v$ is a solution of the following problem:

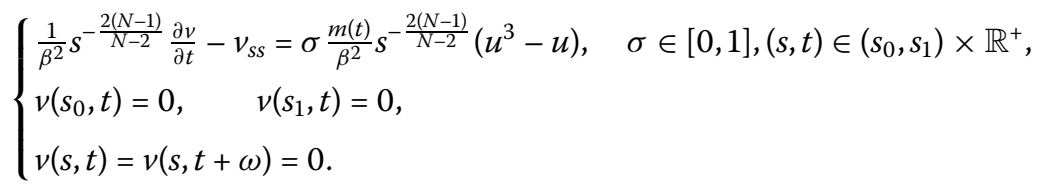

It is easy to check that the operator $K_{\sigma}$ is well defined and completely continuous.

We denote by $\lambda_{1}$ the first eigenvalue of $-\Delta$ in $H_{0}^{1}\left(s_{0}, s_{1}\right)$, and denote $\varphi_{1}(s)$ the corresponding eigenfunction, namely

$$
\left\{\begin{array}{l}
-\left(\varphi_{1}\right)_{s s}=\lambda_{1} \varphi_{1}, \quad s \in\left(s_{0}, s_{1}\right) \\
\varphi_{1}\left(s_{0}\right)=\varphi_{1}\left(s_{1}\right)=0
\end{array}\right.
$$

where $\varphi_{1}(s)>0$.

In the following, we are going to establish the existence of nontrivial time periodic solutions of the problem (5.1) by calculating the topological degree. For this purpose, we denote the ball in $L_{\omega}^{\infty}\left(\left[s_{0}, s_{1}\right] \times[0, \omega]\right)$ with center zero and radius $\widehat{R}$ by $B_{\widehat{R}}$. We first calculate $\operatorname{deg}\left(I-K_{1}, B_{\hat{r}}, 0\right)$ for $\hat{r}$ appropriately small.

Proposition 5.1 $\operatorname{deg}\left(I-K_{1}, B_{\hat{r}}, 0\right)=1$.

Proof We argue by contradiction to prove that $K_{\sigma}$ has no fixed points on $\partial B_{\hat{r}}$, namely

$$
K_{\sigma} v \neq v, \quad \sigma \in[0,1], v \in \partial B_{\hat{r}} .
$$

If $\sigma=0$, then $K_{0}$ is the null operator. Thus

$$
K_{0}(v)=0 \neq v, \quad v \in \partial B_{\hat{r}},
$$

so (5.4) holds. If $0<\sigma \leq 1$, suppose that operator $K_{\sigma}$ admits fixed points on $\partial B_{\hat{r}}$ for some $\sigma \in(0,1]$. Replacing $u$ by $v$ for the first equation of (5.2), we obtain

$$
\frac{1}{\beta^{2}} s^{-\frac{2(N-1)}{N-2}} \frac{\partial v}{\partial t}=v_{s s}+\sigma \frac{m(t)}{\beta^{2}} s^{-\frac{2(N-1)}{N-2}}\left(v^{3}-v\right) .
$$

We choose a constant $\hat{r}$ appropriately small, such that

$$
0<\hat{r}<\left(\frac{\lambda_{1} \beta^{2} s_{0}^{\frac{2(N-1)}{N-2}}}{M}\right)^{\frac{1}{2}}
$$


where $\lambda_{1}$ is defined in (5.3). Multiplying (5.5) by $v$, integrating over $\left[s_{0}, s_{1}\right] \times[0, \omega]$ and using (5.6), by the time periodicity of $v$, we have

$$
\begin{aligned}
0= & \int_{0}^{\omega} \int_{s_{0}}^{s_{1}} \frac{1}{\beta^{2}} s^{-\frac{2(N-1)}{N-2}} \frac{\partial v}{\partial t} v d s d t \\
= & -\int_{0}^{\omega} \int_{s_{0}}^{s_{1}}\left|v_{s}\right|^{2} d s d t+\sigma \int_{0}^{\omega} \int_{s_{0}}^{s_{1}} \frac{m(t)}{\beta^{2}} s^{-\frac{2(N-1)}{N-2}} v^{4} d s d t \\
& -\sigma \int_{0}^{\omega} \int_{s_{0}}^{s_{1}} \frac{m(t)}{\beta^{2}} s^{-\frac{2(N-1)}{N-2}} v^{2} d s d t \\
\leq & \left(\frac{M \hat{r}^{2}}{s_{0}^{-\frac{2(N-1)}{N-2}}}-\lambda_{1}\right) \int_{0}^{\omega} \int_{s_{0}}^{s_{1}} v^{2} d s d t \\
< & 0,
\end{aligned}
$$

which is a contradiction. Therefore, $K_{\sigma}$ has no fixed points on $\partial B_{\hat{r}}$, so (5.4) holds.

On the other hand, since $K_{0}=0$, using the homotopy invariance of the Leray-Schauder degree, we have

$$
\operatorname{deg}\left(I-K_{1}, B_{\hat{r}}, 0\right)=\operatorname{deg}\left(I-K_{0}, B_{\hat{r}}, 0\right)=\operatorname{deg}\left(I, B_{\hat{r}}, 0\right)=1 .
$$

The proof of this proposition is complete.

Next we investigate the Leray-Schauder degree of $I-T_{l}$ in a large ball $B_{\widehat{R}}$ in $L_{\omega}^{\infty}\left(\left[s_{0}, s_{1}\right] \times\right.$ $[0, \omega])$. Let us now introduce a new family of operators $T_{l}$,

$$
\begin{aligned}
& T_{l}: L_{\omega}^{\infty}\left(\left[s_{0}, s_{1}\right] \times[0, \omega]\right) \times[0,1] \rightarrow L_{\omega}^{\infty}\left(\left[s_{0}, s_{1}\right] \times[0, \omega]\right), \\
& (u, l) \mapsto v .
\end{aligned}
$$

We say that $T_{l} u=v$, if $v$ is a solution of the following problem:

$$
\left\{\begin{array}{l}
\frac{1}{\beta^{2}} s^{-\frac{2(N-1)}{N-2}} \frac{\partial v}{\partial t}-v_{s s}=\frac{m(t)}{\beta^{2}} s^{-\frac{2(N-1)}{N-2}}\left(u^{3}-u\right)+(1-l)\left(\frac{m(t)}{\beta^{2}} s^{-\frac{2(N-1)}{N-2}} u+\lambda_{1} u\right) \\
v\left(s_{0}, t\right)=0, \quad v\left(s_{1}, t\right)=0 \\
v(s, t)=v(s, t+\omega)=0, \quad(s, t) \in\left(s_{0}, s_{1}\right) \times \mathbb{R}^{+}
\end{array}\right.
$$

where $\lambda_{1}$ is defined in (5.3). Obviously, we can deduce that $T_{l}$ is well defined and completely continuous.

For $l \in[0,1]$, a fixed point $v$ of the operator $T_{l}$ is the solution of the following problem:

$$
\left\{\begin{array}{l}
\frac{1}{\beta^{2}} s^{-\frac{2(N-1)}{N-2}} \frac{\partial v}{\partial t}-v_{s s}=\frac{m(t)}{\beta^{2}} s^{-\frac{2(N-1)}{N-2}}\left(v^{3}-v\right)+(1-l)\left(\frac{m(t)}{\beta^{2}} s^{-\frac{2(N-1)}{N-2}} v+\lambda_{1} v\right), \\
v\left(s_{0}, t\right)=0, \quad v\left(s_{1}, t\right)=0 \\
v(s, t)=v(s, t+\omega)=0, \quad(s, t) \in\left(s_{0}, s_{1}\right) \times \mathbb{R}^{+}
\end{array}\right.
$$

Proposition 5.2 If $l=0$, then the problem (5.7) does not admit nontrivial periodic solutions. 
Proof Multiplying the first equation of the problem (5.7) by $\varphi_{1}(s)$, where $\varphi_{1}(s)$ is defined in (5.3), integrating over $\left[s_{0}, s_{1}\right] \times[0, \omega]$, by the periodicity of $v$, we obtain

$$
\begin{aligned}
0 & =\int_{0}^{\omega} \int_{s_{0}}^{s_{1}} v_{s s} \varphi_{1}(s) d s d t+\int_{0}^{\omega} \int_{s_{0}}^{s_{1}} \frac{m(t)}{\beta^{2}} s^{-\frac{2(N-1)}{N-2}} v^{3} \varphi_{1}(s)+\lambda_{1} v \varphi_{1}(s) d s d t \\
& =\underbrace{\int_{0}^{\omega} \int_{s_{0}}^{s_{1}} v\left(\varphi_{1}\right)_{s s}+\lambda_{1} v \varphi_{1}(s) d s d t}_{0}+\int_{0}^{\omega} \int_{s_{0}}^{s_{1}} \frac{m(t)}{\beta^{2}} s^{-\frac{2(N-1)}{N-2}} v^{3} \varphi_{1}(s) d s d t \\
& >0,
\end{aligned}
$$

which is a contradiction. The proof of this proposition is complete.

Next we consider the existence of the solutions for the problem (5.7) when $l \in(0,1]$.

Lemma 5.1 For $N \geq 3$, if $v$ is a positive solution of the problem (5.7), then there exists a positive constant $C$, which is independent of $l$, $v$, such that

$$
\|v\|_{L^{3}\left(\left[s_{0}, s_{1}\right] \times[0, \omega]\right)}^{3}=\int_{0}^{\omega} \int_{s_{0}}^{s_{1}} v^{3} d x d t \leq C .
$$

Proof From the first equation of (5.7), for $l \in(0,1]$, we obtain

$$
\frac{s^{\frac{-2(N-1)}{N-2}}}{\beta^{2}} \frac{\partial v}{\partial t}-v_{s s}=\frac{m(t) s^{\frac{-2(N-1)}{N-2}}}{\beta^{2}} v^{3}-\left(\frac{m(t) s^{\frac{-2(N-1)}{N-2}}}{\beta^{2}}+\lambda_{1}\right) l v+\lambda_{1} v .
$$

Multiplying (5.8) by $\varphi_{1}(s)$, using the periodicity of $v$, we have

$$
\begin{aligned}
& \underbrace{\int_{0}^{\omega} \int_{s_{0}}^{s_{1}} \frac{s^{\frac{-2(N-1)}{N-2}}}{\beta^{2}} \frac{\partial v}{\partial t} \varphi_{1}(s) d s d t}_{0}-\int_{0}^{\omega} \int_{s_{0}}^{s_{1}} v_{s s} \varphi_{1}(s) d s d t \\
& =\int_{0}^{\omega} \int_{s_{0}}^{s_{1}}\left(\frac{m(t) s^{-\frac{2(N-1)}{N-2}}}{\beta^{2}} v^{3} \varphi_{1}(s)\right. \\
& \left.\quad-\left(\frac{m(t) s^{\frac{-2(N-1)}{N-2}}}{\beta^{2}}+\lambda_{1}\right) l v \varphi_{1}(s)+\lambda_{1} v \varphi_{1}(s)\right) d s d t .
\end{aligned}
$$

Using (5.3), we obtain

$$
-\int_{0}^{\omega} \int_{s_{0}}^{s_{1}} v_{s s} \varphi_{1}(s) d s d t=-\int_{0}^{\omega} \int_{s_{0}}^{s_{1}} v\left(\varphi_{1}(s)\right)_{s s} d s d t=\int_{0}^{\omega} \int_{s_{0}}^{s_{1}} \lambda_{1} v \varphi_{1}(s) d s d t .
$$

Substituting (5.10) into (5.9), we have

$$
\begin{aligned}
& \int_{0}^{\omega} \int_{s_{0}}^{s_{1}} \frac{m(t) s^{-\frac{2(N-1)}{N-2}}}{\beta^{2}} v^{3} \varphi_{1}(s) d s d t \\
& \quad=l \int_{0}^{\omega} \int_{s_{0}}^{s_{1}}\left(\frac{m(t) s^{\frac{-2(N-1)}{N-2}}}{\beta^{2}}+\lambda_{1}\right) v \varphi_{1}(s) d s d t, \quad l \in(0,1] .
\end{aligned}
$$


We shall use the fact that if $\Omega$ is of class $C^{2}$, then there exist constants $c_{1}, c_{2}>0$, such that

$$
c_{1} \operatorname{dist}(x, \partial \Omega) \leq \varphi_{1}(x) \leq c_{2} \operatorname{dist}(x, \partial \Omega), \quad x \in \Omega
$$

This is a consequence of $u \in C^{1}(\bar{\Omega})$ and of Hopf's lemma, $c f$. Proposition 52.1(iii) in [29]. Since the annulus domain $\Omega=B_{R_{0}} \backslash B_{r_{0}}, s_{0}=R_{0}^{-\beta}, s_{1}=r_{0}^{-\beta}, 0<s_{0}<s<s_{1}$, using (5.12), we have

$$
\max \left\{c_{2} \operatorname{dist}\left(s, s_{0}\right), c_{2} \operatorname{dist}\left(s, s_{1}\right)\right\} \leq c_{2}\left(s_{1}-s_{0}\right) .
$$

By the assumption $\left(\mathrm{H}_{1}\right), 0<m_{0} \leq m(t) \leq M$. Using Hölder's inequality, (5.11), and (5.13), we infer

$$
\begin{aligned}
& \frac{m_{0} s_{1}^{-\frac{2(N-1)}{N-2}}}{\beta^{2}} \int_{0}^{\omega} \int_{s_{0}}^{s_{1}} v^{3} \varphi_{1}(s) d s d t \\
& \leq \int_{0}^{\omega} \int_{s_{0}}^{s_{1}} \frac{m(t) s^{-\frac{2(N-1)}{N-2}}}{\beta^{2}} v^{3} \varphi_{1}(s) d s d t \\
& =l \int_{0}^{\omega} \int_{s_{0}}^{s_{1}}\left(\frac{m(t) \frac{-2(N-1)}{N-2}}{\beta^{2}}+\lambda_{1}\right) v \varphi_{1}(s) d s d t \\
& \leq\left(\frac{M s_{0}^{\frac{-2(N-1)}{N-2}}}{\beta^{2}}+\lambda_{1}\right) \int_{0}^{\omega} \int_{s_{0}}^{s_{1}}\left(v \varphi_{1}^{\frac{1}{3}}\right) \varphi_{1}^{\frac{2}{3}} d s d t \\
& \leq\left(\frac{M s_{0}^{\frac{-2(N-1)}{N-2}}}{\beta^{2}}+\lambda_{1}\right)\left(\int_{0}^{\omega} \int_{s_{0}}^{s_{1}} v^{3} \varphi_{1} d s d t\right)^{\frac{1}{3}}\left(\int_{0}^{\omega} \int_{s_{0}}^{s_{1}} \varphi_{1} d s d t\right)^{\frac{2}{3}} \\
& \leq\left(\frac{M s_{0}}{\beta^{2}}+\lambda_{1}\right)\left(\int_{0}^{\omega} \int_{s_{0}}^{s_{1}} v^{3} \varphi_{1} d s d t\right)^{\frac{1}{3}}\left(\int_{0}^{\omega} \int_{s_{0}}^{s_{1}} c_{2}\left(s_{1}-s_{0}\right) d s d t\right)^{\frac{2}{3}} .
\end{aligned}
$$

From the above inequality, we have

$$
\int_{0}^{\omega} \int_{s_{0}}^{s_{1}} v^{3} \varphi_{1}(s) d s d t \leq C
$$

We fix a sufficiently small positive constant $\varepsilon_{0}$, from (5.12), we infer there exists a constant $C_{\varepsilon_{0}}>0$, such that

$$
\varphi_{1}(s) \geq C_{\varepsilon_{0}}, \quad s \in\left(s_{0}+\varepsilon_{0}, s_{1}-\varepsilon_{0}\right) .
$$

We deduce from (5.14) and (5.15) that

$$
\begin{aligned}
C_{\varepsilon_{0}} \int_{0}^{\omega} \int_{s_{0}+\varepsilon_{0}}^{s_{1}-\varepsilon_{0}} v^{3} d s d t & \leq \int_{0}^{\omega} \int_{s_{0}+\varepsilon_{0}}^{s_{1}-\varepsilon_{0}} v^{3} \varphi_{1}(s) d s d t \\
& \leq \int_{0}^{\omega} \int_{s_{0}}^{s_{1}} v^{3} \varphi_{1}(s) d s d t \\
& \leq C .
\end{aligned}
$$


Using Lemma 4.2 and (5.16), we obtain

$$
\|v\|_{L^{3}\left(\left[s_{0}, s_{1}\right] \times[0, \omega]\right)}^{3}=\int_{0}^{\omega} \int_{s_{0}}^{s_{1}} v^{3} d s d t \leq C .
$$

In order to calculate $\operatorname{deg}\left(I-T_{l}, B_{\widehat{R}}, 0\right)$ for appropriately large $\widehat{R}$, we need the following maximum norm estimates. Due to Proposition 5.2, we consider $l \in(0,1]$ now.

Lemma 5.2 For $N \geq 3, l \in(0,1]$, if $v$ is a solution of the problem (5.7), then there exists a positive constant $\widehat{C}$, which is independent of $l, v$, such that

$$
\|v\|_{L_{\omega}^{\infty}\left(\left[s_{0}, s_{1}\right] \times[0, \omega]\right)} \leq \widehat{C} .
$$

Proof We will prove the boundedness of $v$ in $W_{\omega}^{1,1 ; 2}\left(\left[s_{0}, s_{1}\right] \times[0, \omega]\right)$. Then we use the Sobolev embedding theorem and the bootstrap argument to obtain $L_{\omega}^{\infty}\left(\left[s_{0}, s_{1}\right] \times[0, \omega]\right)$ estimates. Multiplying (5.8) by $v$, integrating over $\left[s_{0}, s_{1}\right] \times[0, \omega]$, and using the periodicity of $v$, we obtain

$$
\begin{aligned}
\int_{0}^{\omega} & \int_{s_{0}}^{s_{1}}\left|v_{s}\right|^{2} d s d t \\
& =\underbrace{\int_{0}^{\omega} \int_{s_{0}}^{s_{1}} \frac{s^{\frac{-2(N-1)}{N-2}}}{\beta^{2}} \frac{\partial v}{\partial t} v d s d t}_{0}-\int_{0}^{\omega} \int_{s_{0}}^{s_{1}} v v_{s s} d s d t \\
& =\int_{0}^{\omega} \int_{s_{0}}^{s_{1}} \frac{m(t) s^{-\frac{2(N-1)}{N-2}}}{\beta^{2}} v^{4}-\left(\frac{m(t) s^{\frac{-2(N-1)}{N-2}}}{\beta^{2}}+\lambda_{1}\right) l v^{2}+\lambda_{1} v^{2} d s d t .
\end{aligned}
$$

By the assumption $\left(\mathrm{H}_{1}\right)$, we have $0<m_{0} \leq|m(t)| \leq M$. Using (5.18) and Young's inequality, we have

$$
\begin{aligned}
& \int_{0}^{\omega} \int_{s_{0}}^{s_{1}}\left|v_{s}\right|^{2} d s d t \\
& \quad \leq \int_{0}^{\omega} \int_{s_{0}}^{s_{1}} \frac{m(t) s^{-\frac{2(N-1)}{N-2}}}{\beta^{2}} v^{4}+\lambda_{1} v^{2} d s d t \\
& \quad \leq \frac{M s_{0}^{-\frac{2(N-1)}{N-2}}}{\beta^{2}} \int_{0}^{\omega} \int_{s_{0}}^{s_{1}} v^{4} d s d t+\int_{0}^{\omega} \int_{s_{0}}^{s_{1}} \frac{\left(\lambda_{1}\right)^{2}}{2}+\frac{\left(v^{2}\right)^{2}}{2} d s d t \\
& \quad \leq C\|v\|_{L^{4}\left(\left[s_{0}, s_{1}\right] \times[0, \omega]\right)}^{4}
\end{aligned}
$$

From the first equation of (5.7), we obtain

$$
\frac{s^{\frac{-2(N-1)}{N-2}}}{\beta^{2}} \frac{\partial v}{\partial t}-v_{s s}=\frac{s^{\frac{-2(N-1)}{N-2}}}{\beta^{2}} m(t)\left(v^{3}-l v\right)+(1-l) \lambda_{1} v .
$$

Since $v\left(s_{0}, t\right)=v\left(s_{1}, t\right)=0$, we have

$$
v_{t}\left(s_{0}, t\right)=v_{t}\left(s_{1}, t\right)=0 .
$$


Using the periodicity of $v$ and (5.21), we obtain

$$
\begin{aligned}
& \int_{0}^{\omega} \int_{s_{0}}^{s_{1}} v_{s s} v_{t} d s d t \\
& \quad=\underbrace{\left.\int_{0}^{\omega}\left((\nabla v \cdot \vec{n}) v_{t}\right)\right|_{s=s_{0}} ^{s=s_{1}}}_{0} d t-\int_{0}^{\omega} \int_{s_{0}}^{s_{1}} v_{s} v_{s t} d s d t \\
& =-\frac{1}{2} \int_{0}^{\omega} \int_{s_{0}}^{s_{1}} \frac{\partial}{\partial t} v_{s}^{2} d s d t \\
& =0 .
\end{aligned}
$$

Multiplying (5.20) by $v_{t}$, using (5.22), we have

$$
\begin{aligned}
& \int_{0}^{\omega} \int_{s_{0}}^{s_{1}} \frac{s^{\frac{-2(N-1)}{N-2}}}{\beta^{2}}\left|v_{t}\right|^{2} d s d t-\underbrace{\int_{0}^{\omega} \int_{s_{0}}^{s_{1}} v_{s s} v_{t} d s d t}_{0} \\
& =\int_{0}^{\omega} \int_{s_{0}}^{s_{1}} \frac{s^{\frac{-2(N-1)}{N-2}}}{\beta^{2}} m(t)\left(\left(v^{3}-l v\right) v_{t}\right)+(1-l)\left(\lambda_{1} v v_{t}\right) d s d t \\
& =\int_{0}^{\omega} \int_{s_{0}}^{s_{1}} \frac{s^{\frac{-2(N-1)}{N-2}}}{\beta^{2}} m(t) \frac{\partial\left(\frac{v^{4}}{4}-\frac{l v^{2}}{2}\right)}{\partial t} d s d t+\underbrace{\int_{0}^{\omega} \int_{s_{0}}^{s_{1}}(1-l) \frac{\partial\left(\frac{\lambda_{1} v^{2}}{2}\right)}{\partial t} d s d t}_{0} \\
& =-\int_{0}^{\omega} \int_{s_{0}}^{s_{1}} \frac{s^{\frac{-2(N-1)}{N-2}}}{\beta^{2}} m^{\prime}(t)\left(\frac{v^{4}}{4}-\frac{l v^{2}}{2}\right) d s d t .
\end{aligned}
$$

By the assumption $\left(\mathrm{H}_{1}\right)$, we have $0 \leq\left|m^{\prime}(t)\right| \leq C$. Using (5.23) and Young's inequality, we obtain

$$
\begin{aligned}
& \frac{s_{1}^{\frac{-2(N-1)}{N-2}}}{\beta^{2}} \int_{0}^{\omega} \int_{s_{0}}^{s_{1}}\left|v_{t}\right|^{2} d s d t \\
& \leq \int_{0}^{\omega} \int_{s_{0}}^{s_{1}} \frac{s^{\frac{-2(N-1)}{N-2}}}{\beta^{2}}\left|v_{t}\right|^{2} d s d t \\
& \leq \frac{s_{0}^{\frac{-2(N-1)}{N-2}}}{\beta^{2}} \int_{0}^{\omega} \int_{s_{0}}^{s_{1}}\left|v_{t}\right|^{2} d s d t \\
& \leq \frac{s_{0}^{\frac{-2(N-1)}{N-2}}}{\beta^{2}} \int_{0}^{\omega} \int_{s_{0}}^{s_{1}} \frac{s^{\frac{-2(N-1)}{N-2}}}{\beta^{2}}\left|-m^{\prime}(t)\left(\frac{v^{4}}{4}-\frac{l v^{2}}{2}\right)\right| d s d t \\
& \leq C\|v\|_{L^{4}\left(\left[s_{0}, s_{1}\right] \times[0, \omega]\right)}^{\cdot}
\end{aligned}
$$

Combining the above inequality with (5.19), we have

$$
\begin{aligned}
\|v\|_{W_{\omega}^{1,1 ; 2}\left(\left[s_{0}, s_{1}\right] \times[0, \omega]\right)}^{2} & =\int_{0}^{\omega} \int_{s_{0}}^{s_{1}}\left|v_{s}\right|^{2} d s d t+\int_{0}^{\omega} \int_{s_{0}}^{s_{1}}\left|v_{t}\right|^{2} d s d t \\
& \leq C\|v\|_{L^{4}\left(\left[s_{0}, s_{1}\right] \times[0, \omega]\right)}^{4} .
\end{aligned}
$$


By the interpolation inequality for $L^{4}\left(\left[s_{0}, s_{1}\right] \times[0, \omega]\right)$ norms, using Lemma 5.1 , we have

$$
\|v\|_{4}^{4} \leq\left(\|v\|_{3}^{\theta}\|v\|_{\hat{q}}^{1-\theta}\right)^{4} \leq C\|v\|_{\hat{q}}^{4(1-\theta)}, \quad \theta \in(0,1)
$$

where $\hat{q}>4$, and

$$
\frac{1}{4}=\frac{\theta}{3}+\frac{1-\theta}{\hat{q}}, \quad \theta=\frac{3(\hat{q}-4)}{4(\hat{q}-3)} .
$$

Using the Sobolev embedding theorem, we obtain

$$
\|v\|_{L^{\hat{q}\left(\left[s_{0}, s_{1}\right] \times[0, \omega]\right)}}^{\hat{q}} \leq C\|v\|_{W_{\omega}^{1,1 ; 2}\left(\left[s_{0}, s_{1}\right] \times[0, \omega]\right)}^{2}, \quad \hat{q}>4
$$

Combining (5.24), (5.25), and (5.26), using Young's inequality, we have

$$
\begin{aligned}
\|v\|_{W_{\omega}^{1,1 ; 2}\left(\left[s_{0}, s_{1}\right] \times[0, \omega]\right)}^{2} & \leq C\|v\|_{L^{4}\left(\left[s_{0}, s_{1}\right] \times[0, \omega]\right)}^{4} \\
\leq & C\|v\|_{\hat{q}}^{4(1-\theta)} \\
& =C\left(\|v\|_{\hat{q}}^{\hat{q}}\right)^{\frac{4(1-\theta)}{\hat{q}}} \\
\leq & C\left(C\|v\|_{W_{\omega}^{1,1 ; 2}\left(\left[s_{0}, s_{1}\right] \times[0, \omega]\right)}^{2}\right)^{\frac{4(1-\theta)}{\hat{q}}} \\
\leq & \frac{\varepsilon\left(\left(\|v\|_{W_{\omega}^{1,1 ; 2}\left(\left[s_{0}, s_{1}\right] \times[0, \omega]\right)}^{2}\right)^{\frac{4(1-\theta)}{\hat{q}}}\right)^{\eta}}{\eta}+\frac{\varepsilon^{\frac{1}{1-\eta}}\left(C C^{\frac{4(1-\theta)}{\hat{q}}}\right)^{\frac{\eta}{\eta-1}}}{\frac{\eta}{\eta-1}} \\
\leq & \varepsilon\|v\|_{W_{\omega}^{1,1 ; 2}\left(\left[s_{0}, s_{1}\right] \times[0, \omega]\right)}^{2}+C,
\end{aligned}
$$

where $\eta:=\frac{\hat{q}}{4(1-\theta)}, \hat{q}>4,0<\theta<1$, thus $\eta>1$. We choose $0<\varepsilon<1, \varepsilon$ sufficiently small. From (5.27), we obtain

$$
\|v\|_{W_{\omega}^{1,1 ; 2}\left(\left[s_{0}, s_{1}\right] \times[0, \omega]\right)}^{2} \leq C
$$

where the constant $C$ is independent of $l, v$. Using the Sobolev embedding theorem and (5.28), we infer $v$ is bounded in $L_{\omega}^{\infty}\left(\left[s_{0}, s_{1}\right] \times[0, \omega]\right)$.

Proposition $5.3 \operatorname{deg}\left(I-K_{1}, B_{\widehat{R}}, 0\right)=0$.

Proof In fact, if $v$ is a solution of the problem (5.7), then Lemma 5.2 implies that $v$ is bounded in $L_{\omega}^{\infty}\left(\left[s_{0}, s_{1}\right] \times[0, \omega]\right)$. Thus there exists a constant $\widehat{R}>\min \{\hat{r}, \widehat{C}\}$, where $\hat{r}$ is defined in (5.6), $\widehat{R}$ is independent of $l, v$, such that

$$
\|v\|_{L_{\omega}^{\infty}\left(\left[s_{0}, s_{1}\right] \times[0, \omega]\right)} \leq \widehat{C}<\widehat{R}
$$

From (5.29) we infer $T_{l}$ has no fixed point for $v \in \partial B_{\widehat{R}}$, namely

$$
T_{l} v \neq v, \quad l \in[0,1], \quad\|v\|_{L_{\omega}^{\infty}\left(\left[s_{0}, s_{1}\right] \times[0, \omega]\right)}=\widehat{R} .
$$


For $l=0$, Proposition 5.2 implies the problem (5.7) does not admit nontrivial periodic solutions, thus

$$
\operatorname{deg}\left(I-T_{0}, B_{\widehat{R}}, 0\right)=0
$$

Using the homotopy invariance of degree, from (5.30) we infer

$$
\operatorname{deg}\left(I-T_{1}, B_{\widehat{R}}, 0\right)=\operatorname{deg}\left(I-T_{0}, B_{\widehat{R}}, 0\right)=0 .
$$

We notice that the operator $K_{1}=T_{1}$, then

$$
\operatorname{deg}\left(I-K_{1}, B_{\widehat{R}}, 0\right)=\operatorname{deg}\left(I-T_{1}, B_{\widehat{R}}, 0\right)=0 .
$$

The proof of this proposition is complete.

\section{Proof of the main results}

In this section, we give the proof of the main results of this paper. The following wellknown lemma will be used in our proof.

Lemma 6.1 (Kronecker's existence theorem) Let X be a real normed linear space, $\Omega$ be a bounded open subset of $X$, and $F=I-K$ be a completely continuous field defined on $\bar{\Omega}$, $y \in X \backslash F(\partial \Omega)$. If $y \notin F(\bar{\Omega})$, then $\operatorname{deg}(F, \Omega, y)=0$. Thus, if $\operatorname{deg}(F, \Omega, y) \neq 0$, then the equation $F(x)=y$ admits at least one solution in $\Omega$.

Proof of Theorem 2.1 Combining Proposition 3.1 and Proposition 4.1, we have

$$
\operatorname{deg}\left(I-K_{1}, B_{R}-B_{r}, 0\right)=\operatorname{deg}\left(I-K_{1}, B_{R}, 0\right)-\operatorname{deg}\left(I-K_{1}, B_{r}, 0\right)=-1 .
$$

By Lemma 6.1, we know that the problem (1.1)-(1.3) admits a nontrivial periodic solution $u \in L_{\omega}^{\infty}\left(Q_{\omega}\right)$. Using a standard bootstrap procedure, we can improve the regularity of the solution, and we conclude that the problem (1.1)-(1.3) admits a nontrivial classical periodic solution $u \in C_{\omega}^{2+\alpha, 1+\frac{\alpha}{2}}\left(Q_{\omega}\right)$ for some $\alpha \in(0,1)$. The proof of this theorem is complete.

Proof of Theorem 2.2 We argue by contradiction. Assume that the problem (1.1)-(1.3) admits a positive periodic solution $u$. For any fixed $\xi \in[\tau, \tau+\omega]$, multiplying equation (1.1) by $x \cdot \nabla u(x, \xi)$ on both sides, and integrating over $\Omega$, we obtain

$$
\begin{gathered}
\int_{\Omega} u_{t}(x \cdot \nabla u(x, \xi)) d x-\int_{\Omega} \Delta u(x, t)(x \cdot \nabla u(x, \xi)) d x \\
=\int_{\Omega} m(t)\left(u^{3}(x, t)-u(x, t)\right)(x \cdot \nabla u(x, \xi)) d x .
\end{gathered}
$$

For simplicity, we denote the first term of the left side of (6.1) by $J_{1}(t)$. By the periodicity of $u$, we get

$$
\int_{\tau}^{\tau+\omega} \int_{\Omega} u_{t}(x \cdot \nabla u(x, \xi)) d x d t=\int_{\tau}^{\tau+\omega} J_{1}(t) d t=0
$$


By the integral mean value theorem and (6.2), there exists a $t \in[\tau, \tau+\omega]$, such that $J_{1}(t)=0$. Similar to the proof of [21], p.620, we denote $t_{\xi}=\min \left\{t \in[\tau, \tau+\omega] ; J_{1}(t)=0\right\}$. Define an operator

$$
F:[\tau, \tau+\omega] \rightarrow[\tau, \tau+\omega], \quad F(\xi) \mapsto t_{\xi} .
$$

By $F(\tau)=t_{\tau} \geq \tau$, we have $F(\tau)-\tau \geq 0$. By $F(\tau+\omega)=t_{\tau+\omega} \leq \tau+\omega$, we have $F(\tau+\omega)-(\tau+$ $\omega) \leq 0$. Thus there exists a $t^{*} \in[\tau, \tau+\omega]$ such that $F\left(t^{*}\right)=t^{*}$. By (6.1), we have

$$
\begin{aligned}
& \underbrace{J_{1}\left(t^{*}\right)}_{0}-\int_{\Omega} \Delta u\left(x, t^{*}\right)\left(x \cdot \nabla u\left(x, t^{*}\right)\right) d x \\
& \quad=\int_{\Omega} m\left(t^{*}\right)\left(u^{3}\left(x, t^{*}\right)-u\left(x, t^{*}\right)\right)\left(x \cdot \nabla u\left(x, t^{*}\right)\right) d x .
\end{aligned}
$$

We proceed in a similar way to (4.13)-(4.14). Similar to (4.14) with $L=0$, we obtain

$$
\begin{aligned}
0= & N \int_{\Omega} m\left(t^{*}\right)\left(\frac{u^{4}\left(x, t^{*}\right)}{4}-\frac{u^{2}\left(x, t^{*}\right)}{2}\right) d x \\
& +\int_{\Omega} m\left(t^{*}\right)\left(u^{3}\left(x, t^{*}\right)-u\left(x, t^{*}\right)\right)\left(x \cdot \nabla u\left(x, t^{*}\right)\right) d x .
\end{aligned}
$$

For the fixed $t^{*}$, we have $u_{t}\left(x, t^{*}\right)=0$. Multiplying the following equation:

$$
\underbrace{u_{t}\left(x, t^{*}\right)}_{0}-\Delta u\left(x, t^{*}\right)=m\left(t^{*}\right)\left(u^{3}\left(x, t^{*}\right)-u\left(x, t^{*}\right)\right)
$$

by $u\left(x, t^{*}\right)$ on both sides, and integrating over $\Omega$, we proceed in a similar way to (4.15)(4.16). Similar to (4.16) with $L=0$, we obtain

$$
\begin{aligned}
& \int_{\Omega} \Delta u\left(x, t^{*}\right)\left(x \cdot \nabla u\left(x, t^{*}\right)\right) d x \\
& \quad=\frac{(N-2)}{2} \int_{\Omega} m\left(t^{*}\right)\left(u^{4}\left(x, t^{*}\right)-u^{2}\left(x, t^{*}\right)\right) d x+\frac{1}{2} \int_{\partial \Omega}\left|\nabla u\left(x, t^{*}\right)\right|^{2}(x \cdot \vec{n}) d s .
\end{aligned}
$$

Substituting (6.4) and (6.5) into (6.3), we have

$$
\begin{aligned}
& \int_{\Omega}\left(\left(1-\frac{N}{4}\right) u^{4}\left(x, t^{*}\right)\right) m\left(t^{*}\right) d x \\
& =\int_{\Omega} u^{2}\left(x, t^{*}\right) m\left(t^{*}\right) d x+\frac{1}{2} \int_{\partial \Omega}\left|\nabla u\left(x, t^{*}\right)\right|^{2}(x \cdot \vec{n}) d s .
\end{aligned}
$$

The nontrivial periodic solution of problem (1.1)-(1.3) implies that there exists a point $x^{*} \in \Omega$ such that $u\left(x^{*}, t^{*}\right) \neq 0$. Since $\Omega$ is star shaped, we have

$$
(x \cdot \vec{n}) \geq 0, \quad x \in \partial \Omega,
$$

where $\vec{n}$ denotes the unit outward normal vector at $x \in \partial \Omega$. Due to $m(t)$ being a positive $\omega$ periodic function, for $N \geq 4$, the left side of equality (6.6) is less than zero or equal to zero. 
But the right-hand side of equality (6.6) is strictly greater than zero. This contradiction implies that there is no nontrivial periodic solution for the problem (1.1)-(1.3). The proof of this theorem is complete.

Proof of Theorem 2.3 Combining Proposition 5.1 and Proposition 5.3, we obtain

$$
\operatorname{deg}\left(I-K_{1}, B_{\widehat{R}}-B_{\hat{r}}, 0\right)=\operatorname{deg}\left(I-K_{1}, B_{\widehat{R}}, 0\right)-\operatorname{deg}\left(I-K_{1}, B_{\hat{r}}, 0\right)=-1 .
$$

By Lemma 6.1, we know that the problem (5.1) admits nontrivial periodic solutions $v \in$ $L_{\omega}^{\infty}\left(\left[s_{0}, s_{1}\right] \times[0, \omega]\right)$. It means that the problem (1.1)-(1.3) admits a nontrivial radial solution. Using a standard bootstrap procedure, we can improve the regularity of the solution and conclude that the problem (1.1)-(1.3) admits a classical radial periodic solution $u \in C_{\omega}^{2+\alpha, 1+\frac{\alpha}{2}}\left(Q_{\omega}\right)$ for some $\alpha \in(0,1)$. The proof of this theorem is complete.

\section{Numerical simulations}

In this section, we give some numerical simulations to illustrate our results. Especially, we will show how the positive periodic solutions of the problem (1.1)-(1.3) depend on changing $m(t)$. For simplicity, we take $(x, t) \in(0,1) \times(0,4 \pi)$ throughout this section.

We first give a simple corresponding ODE model as follows:

$$
\begin{aligned}
& -v_{x x}=m\left(v^{3}-v\right), \quad x \in(0,1), \\
& v(0)=0, \quad v(1)=0, \quad v(x)>0, \quad x \in(0,1) .
\end{aligned}
$$

In order to use the shooting method, we consider the following auxiliary problem:

$$
\begin{aligned}
& -v_{x x}=m\left(v^{3}-v\right), \quad x \in(0,1), \\
& v(0)=0, \quad v^{\prime}(0)=a, \quad v(x)>0, \quad x \in(0,1),
\end{aligned}
$$

where the positive constant $a$ will be chosen such that $v(1)=0$. If we take $m=0.5$, numerical simulation shows that $v(1)=1.36581 \times 10^{-6}$ by choosing $a=13.9716$, which can be viewed as an approximate solution of (7.1).

Similar to the above process, we choose $m=1,2,3,10$, respectively, and set the allowable error $9 \times 10^{-6}$. Then the corresponding approximate solutions of (7.1) are shown in Figure 1 and the values of $a$ and $v(1)$ are listed as follows:

(i) $m=1, a=10.026, v(1)=4.27318 \times 10^{-6}$,

(ii) $m=2, a=7.27742, v(1)=6.45078 \times 10^{-6}$,

Figure 1 By the shooting method, the numerical solutions for problem (7.1) with $m=0.5,1,2,3$, 10 , respectively.

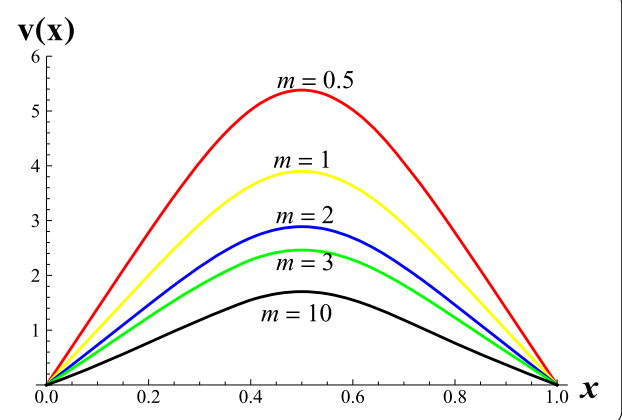


(iii) $m=3, a=6.07622, v(1)=3.06822 \times 10^{-6}$,

(iv) $m=10, a=3.61666, v(1)=3.22153 \times 10^{-7}$.

Now we take time $t$ into account. Obviously, if $m(t) \equiv m$, by (7.1), we can easily construct trivial time-periodic solutions of the problem (1.1)-(1.3). In fact, we can consider the following auxiliary initial boundary value problem:

$$
\begin{aligned}
& u_{t}-u_{x x}=m\left(u^{3}-u\right), \quad x \in(0,1), t \in(0,4 \pi), \\
& u(0)=0, \quad u(1)=0 \\
& u(x, 0)=v(x)
\end{aligned}
$$

where $v(x)$ is the solution of the problem (7.1), $m$ is the same positive constant as the problem (7.1). Then any solution of (7.3) is also a periodic solution of the problem (1.1)(1.3) with $m(t) \equiv m$.

Now we will numerically show that, for the case of $m(t) \not \equiv m$, the story will be totally different. In (7.3), we set $u(x, 0)=\sin (\pi x)+x(x-1)$, and choose two different $m(t)=11+$ $10 \cos (t), m(t)=11+10 \sin (t)$, respectively. Then the numerical solutions of the problem (1.1)-(1.3) decay to 0 (see Figures 2, 3).

If we choose $u(x, 0)=1.07 \sin (\pi x)+1.07 x(x-1), u(x, 0)=1.3 \sin (\pi x)+1.3 x(x-1)$, respectively, then the numerical solutions of the problem (1.1)-(1.3) blow up in finite time (see Figures 4, 5).

Summarizing the numerical simulations, we see that if $m(t) \equiv m$, then a smaller $m$ will give a larger positive periodic solution for the problem (1.1)-(1.3) (see Figure 1). While $m(t) \not \equiv m$, the numerical simulations suggest that the periodic solutions for the problem (1.1)-(1.3) are unstable (see Figures 2, 3, 4, 5).

Figure $2 m(t)=11+10 \cos (t)$ $u(x, 0)=\sin (\pi x)+x(x-1)$.

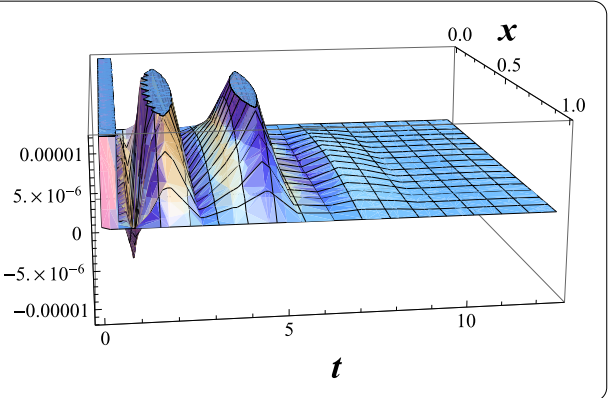

Figure $3 m(t)=11+10 \sin (t)$, $u(x, 0)=\sin (\pi x)+x(x-1)$.

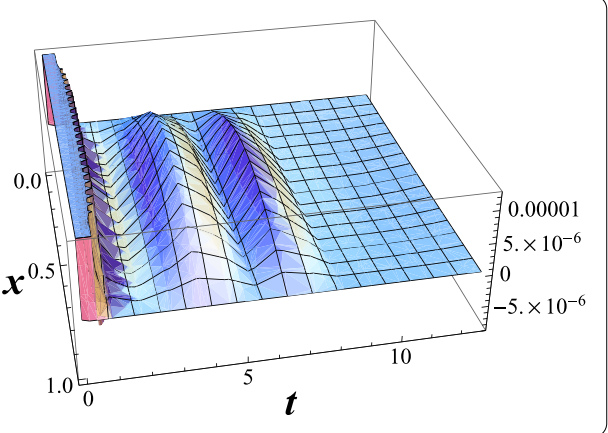




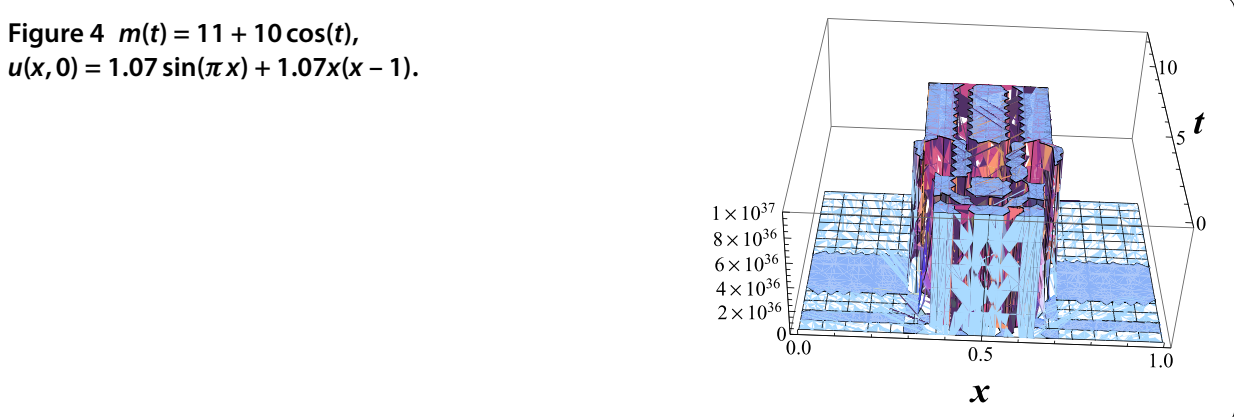

\section{Figure $5 m(t)=11+10 \sin (t)$ \\ $u(x, 0)=1.3 \sin (\pi x)+1.3 x(x-1)$.}

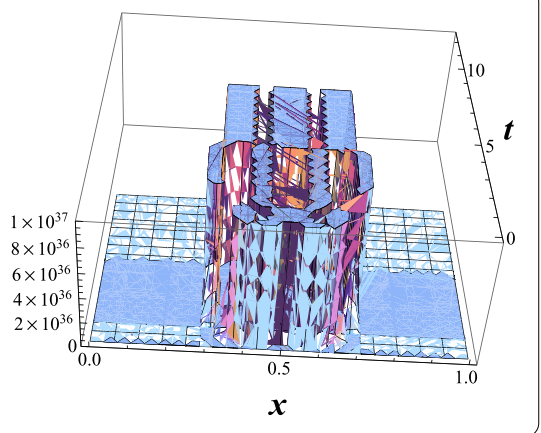

\section{Competing interests}

The authors declare that they have no competing interests.

\section{Authors' contributions}

The authors declare that the study was realized in collaboration with the same responsibility. All authors read and approved the final manuscript.

\section{Author details}

${ }^{1}$ School of Mathematical Sciences, South China Normal University, Guangzhou, 510631, China. ${ }^{2}$ School of Mathematics, South China University of Technology, Guangzhou, 510640, China.

\section{Acknowledgements}

The research of R Huang and J Yin was supported in part by NNSFC (No. 11071099). The research of R Huang was supported in part by CPSF (No. 2015M572301) and the Fundamental Research Funds for the Central Universities. The research of S Ji was supported by the Scientific Research Foundation of Graduate School of South China Normal University (No. 2014bsxm01). The research of H Huang was supported by the Scientific Research Foundation of Graduate School of South China Normal University (No. 2014ssxm06).

\section{Received: 21 May 2015 Accepted: 1 September 2015 Published online: 17 September 2015}

\section{References}

1. Allen, S, Cahn, JW: A microscopic theory for antiphase boundary motion and its application to antiphase domain coarsening. Acta Metall. 27, 1084-1095 (1979)

2. Cirilloa, ENM, lanirob, N, Sciarra, G: Allen-Cahn and Cahn-Hilliard-like equations for dissipative dynamics of saturated porous media. J. Mech. Phys. Solids 61(2), 629-651 (2013)

3. Shen, J, Yang, F: Numerical approximations of Allen-Cahn and Cahn-Hilliard equations. Discrete Contin. Dyn. Syst. 28(4), 1669-1691 (2010)

4. Murray, JD: Mathematical Biology, 2nd edn. Springer, Berlin (1993)

5. Cahn, JW, Novick-Cohen, A: Limiting motion for an Allen-Cahn/Cahn-Hilliard system. In: Free Boundary Problems: Theory and Applications (Zakopane, 1995). Pitman Res. Notes Math. Ser., vol. 363, pp. 89-97. Longman, Harlow (1996)

6. de Mottoni, P, Schatzman, M: Geometrical evolution of developed interfaces. Trans. Am. Math. Soc. 347(5), 1533-1589 (1995)

7. de la Llave, R, Valdinoci, E: Multiplicity results for interfaces of Ginzburg-Landau-Allen-Cahn equations in periodic media. Adv. Math. 215, 379-426 (2007)

8. Kowalczyk, M, Liu, Y, Wei, J: Singly periodic solutions of the Allen-Cahn equation and the Toda lattice. Commun Partial Differ. Equ. 40(2), 329-356 (2015)

9. Matano, H, Nara, M: Large time behavior of disturbed planar fronts in the Allen-Cahn equation. J. Differ. Equ. 251, 3522-3557 (2011)

10. Novaga, M, Valdinoci, E: Multibump solutions and asymptotic expansions for mesoscopic Allen-Cahn type equations ESAIM Control Optim. Calc. Var. 15, 914-933 (2009) 
11. Novaga, M, Valdinoci, E: Bump solutions for the mesoscopic Allen-Cahn equation in periodic media. ESAIM Control Optim. Calc. Var. 40, 37-49 (2011)

12. Gurtin, ME: Generalized Ginzburg-Landau and Cahn-Hilliard equations based on a microforce balance. Physica D 92 178-192 (1996)

13. Esteban, MJ: On periodic solutions of superlinear parabolic problems. Trans. Am. Math. Soc. 293, 171-189 (1986)

14. Esteban, MJ: A remark on the existence of positive periodic solutions of superlinear parabolic problems. Proc. Am. Math. Soc. 102, 131-136 (1988)

15. Húska, J: Periodic solutions in superlinear parabolic problems. Acta Math. Univ. Comen. 19, 19-26 (2002)

16. Húska, J: Periodic solutions in semilinear parabolic problems. Thesis, Comenius University, Slovakia (2002)

17. Hirano, N, Mizoguchi, N: Positive unstable periodic solutions for superlinear parabolic equations. Proc. Am. Math. Soc. $123,1487-1495$ (1995)

18. Quittner, P: Multiple equilibria, periodic solutions and a priori bounds for solutions in superlinear parabolic problems. NoDEA Nonlinear Differ. Equ. Appl. 11, 237-258 (2004)

19. Seidman, TI: Periodic solutions of a nonlinear parabolic equation. J. Differ. Equ. 19, 242-257 (1975)

20. Wang, YF, Yin, JX, Wu, ZQ: Periodic solutions of evolution $p$-Laplacian equations with nonlinear sources. J. Math. Anal. Appl. 219, 76-96 (1998)

21. Yin, JX, Jin, CH: Periodic solutions of the evolutionary p-Laplacian with nonlinear sources. J. Math. Anal. Appl. 368, 604-622 (2010)

22. Zhou, $Q$, Huang, $R, K e, Y$, Wang, YF: Existence of the nontrivial nonnegative periodic solutions for the quasilinear parabolic equation with nonlocal terms. Comput. Math. Appl. 50(8-9), 1293-1302 (2005)

23. Deng, J: Existence of positive radial solutions for a nonlinear elliptic problem in annulus domains. Math. Methods Appl. Sci. 35, 1594-1600 (2012)

24. Bartsch, T, Poláčik, P, Quittner, P: Liouville-type theorems and asymptotic behavior of nodal radial solutions of semilinear heat equations. J. Eur. Math. Soc. 13, 219-247 (2011)

25. Hess, P: Periodic-Parabolic Boundary Value Problems and Positivity. Pitman Research Notes in Mathematics Series, vol. 247. Longman, Harlow (1991)

26. Wang, YF, Yin, JX: Periodic solutions for a class of degenerate parabolic equations with Neumann boundary conditions. Nonlinear Anal., Real World Appl. 12(4), 2069-2076 (2011)

27. Wang, YF, Yin, JX: Coexistence periodic solutions of a doubly nonlinear parabolic system with Neumann boundary conditions. J. Math. Anal. Appl. 396(2), 704-714 (2012)

28. Hameed, RA, Sun, JB, Wu, BY: Existence of periodic solutions of a $p$-Laplacian-Neumann problem. Bound. Value Probl. 2013, 171 (2013)

29. Quittner, P, Souplet, P: Superlinear Parabolic Problems: Blow-up, Global Existence and Steady States. Birkhäuser, Berlin (2007)

30. Quittner, P: Continuity of the blow-up time and a priori bounds for solutions in superlinear parabolic problems. Houst. J. Math. 29, 757-799 (2003)

\section{Submit your manuscript to a SpringerOpen ${ }^{\mathcal{O}}$ journal and benefit from:}

- Convenient online submission

- Rigorous peer review

- Immediate publication on acceptance

- Open access: articles freely available online

- High visibility within the field

- Retaining the copyright to your article 